\title{
Similar patterns of genetic diversity and linkage disequilibrium in Western chimpanzees (Pan troglodytes verus) and humans indicate highly conserved mechanisms of MHC molecular evolution
}

Christelle Vangenot ${ }^{1}$, José Manuel Nunes ${ }^{1,2}$, Gaby M. Doxiadis ${ }^{3}$, Estella S. Poloni ${ }^{1,2}$, Ronald E. Bontrop ${ }^{3}$, Natasja G. de Groot ${ }^{3}$ and Alicia Sanchez-Mazas ${ }^{1,2^{*}}$ (D)

\begin{abstract}
Background: Many species are threatened with extinction as their population sizes decrease with changing environments or face novel pathogenic threats. A reduction of genetic diversity at major histocompatibility complex (MHC) genes may have dramatic effects on populations' survival, as these genes play a key role in adaptive immunity. This might be the case for chimpanzees, the MHC genes of which reveal signatures of an ancient selective sweep likely due to a viral epidemic that reduced their population size a few million years ago. To better assess how this past event affected MHC variation in chimpanzees compared to humans, we analysed several indexes of genetic diversity and linkage disequilibrium across seven MHC genes on four cohorts of chimpanzees and we compared them to those estimated at orthologous HLA genes in a large set of human populations.

Results: Interestingly, the analyses uncovered similar patterns of both molecular diversity and linkage disequilibrium across the seven MHC genes in chimpanzees and humans. Indeed, in both species the greatest allelic richness and heterozygosity were found at loci $A, B, C$ and $D R B 1$, the greatest nucleotide diversity at loci DRB1, DQA1 and DQB1, and both significant global linkage disequilibrium and the greatest proportions of haplotypes in linkage disequilibrium were observed at pairs $D Q A 1 \sim D Q B 1, D Q A 1 \sim D R B 1, D Q B 1 \sim D R B 1$ and $B \sim C$. Our results also showed that, despite some differences among loci, the levels of genetic diversity and linkage disequilibrium observed in contemporary chimpanzees were globally similar to those estimated in small isolated human populations, in contrast to significant differences compared to large populations.

(Continued on next page)
\end{abstract}

\footnotetext{
* Correspondence: alicia.sanchez-mazas@unige.ch

'Laboratory of Anthropology, Genetics and Peopling History, Department of

Genetics and Evolution, Anthropology Unit, University of Geneva, Geneva, Switzerland

${ }^{2}$ Institute of Genetics and Genomics in Geneva (iGE3), University of Geneva, Geneva, Switzerland

Full list of author information is available at the end of the article
}

(c) The Author(s). 2020 Open Access This article is licensed under a Creative Commons Attribution 4.0 International License, which permits use, sharing, adaptation, distribution and reproduction in any medium or format, as long as you give appropriate credit to the original author(s) and the source, provide a link to the Creative Commons licence, and indicate if changes were made. The images or other third party material in this article are included in the article's Creative Commons licence, unless indicated otherwise in a credit line to the material. If material is not included in the article's Creative Commons licence and your intended use is not permitted by statutory regulation or exceeds the permitted use, you will need to obtain permission directly from the copyright holder. To view a copy of this licence, visit http://creativecommons.org/licenses/by/4.0/ The Creative Commons Public Domain Dedication waiver (http://creativecommons.org/publicdomain/zero/1.0/) applies to the data made available in this article, unless otherwise stated in a credit line to the data. 
(Continued from previous page)

Conclusions: We conclude, first, that highly conserved mechanisms shaped the diversity of orthologous MHC genes in chimpanzees and humans. Furthermore, our findings support the hypothesis that an ancient demographic decline affecting the chimpanzee populations - like that ascribed to a viral epidemic - exerted a substantial effect on the molecular diversity of their MHC genes, albeit not more pronounced than that experienced by $H L A$ genes in human populations that underwent rapid genetic drift during humans' peopling history. We thus propose a model where chimpanzees' MHC genes regenerated molecular variation through recombination/gene conversion and/or balancing selection after the selective sweep.

Keywords: MHC, Patr, HLA, Western chimpanzees, Human populations, Nucleotide diversity, Linkage disequilibrium, Selective sweep, Balancing selection, Population bottleneck, Demographic history

\section{Background}

The Major Histocompatibility Complex $(M H C)$ is a family of genes that play a major role in activating adaptive immune responses [1]. Some of these gene families code for transmembrane proteins that protect individuals from viral, bacterial and parasitic infections by presenting pathogen-derived peptides to $\mathrm{T}$ lymphocytes, which subsequently triggers an immune response. The $M H C$ molecular region, called HLA in humans and Patr in chimpanzees, is very similar in these two species as orthologous genes involved in peptide presentation are physically arranged in a comparable way [2-7] (Fig. 1). These genes are organized into two classes that differ from each other based on major structural and functional differences between their corresponding proteins. The molecules expressed (on almost all nucleated cells) by the classical class I genes (named $A, B$ and $C$ ) consist of one $\alpha$ chain, non-covalently bound to a small $\beta 2$-microglobulin chain which is not encoded in the $M H C$ region. The $\alpha 1$ and $\alpha 2$ domains of this heavy chain form the peptide-binding region (PBR) which presents short peptides (mostly nonamers) of intracellular origin at the cell surface to CD8+ cytotoxic $\mathrm{T}$ lymphocytes. In all classical $M H C$ class I genes, the 2nd and 3rd exons encoding these two domains are highly polymorphic. Chimpanzees may also possess an additional class I $A$-like locus named Patr- $A L$ which is in strong linkage disequilibrium with $\operatorname{Patr}-A[8,9]$. However this gene is not fixed but only present on a portion of the haplotypes. The $M H C$ molecules encoded by the class II genes (named $D P, D Q$ and $D R$ ) display a more specific tissue distribution limited to professional antigen presenting cells implicated in the immune response, i.e. mostly $\mathrm{B}$ lymphocytes, dendritic cells and macrophages. Contrary to class I, class II proteins are heterodimers composed of one $\alpha$ chain coded by a " $A$ " gene (named $D P A, D Q A$ or $D R A$ ) and one $\beta$ chain coded by a " $B$ " gene (named $D P B, D Q B$ or $D R B$, respectively). The $\alpha 1$ and $\beta 1$ domains of the $\alpha$ and $\beta$ chains form the PBR, which in this case presents peptides (of about 12-15 amino acids) from mostly extracellular origin at the cell surface to CD4+ T-helper lymphocytes. The 2nd exon of most $M H C$ class II " $B$ " genes (which encodes the $\beta 1$ domain) is highly variable, whereas that of " $A$ " genes (which encodes the $\alpha 1$ domain) is much less polymorphic, except at the $D Q$ loci. Most class II genes also exhibit one or more functional and/or non-functional (i.e. pseudogenic) copies (e.g. $D R B 1, D R B 2, D R B 3$, etc...)

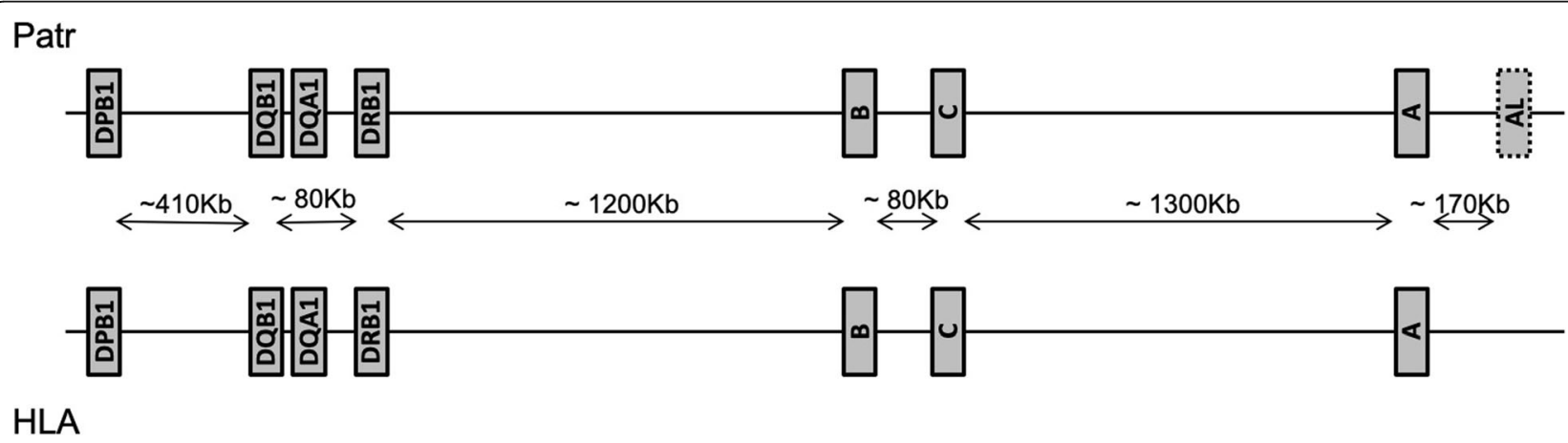

Fig. 1 Map of the human and chimpanzee MHC region showing average physical distances between the 7 loci under study in both species. The distances between loci (in $\mathrm{Kb}=$ kilobases) slightly vary between the two species but they have the same order of magnitude. $\sim 80 \mathrm{~Kb}$ stands for "physical distance between DQB1 and DRB1 is about $80 \mathrm{~Kb}$ " 
resulting from past duplications [5, 10-16], but only the four most polymorphic ones $D P B 1, D Q B 1, D Q A 1$ and $D R B 1$ are extensively studied.

The $H L A$ region is amongst the most variable of the whole genome, with almost 26,000 HLA (class I and class II) alleles identified so far (November 2019, [17]). Its huge level of diversity and/or allelic variation observed within human populations is believed to be maintained by different kinds of balancing selection, most often in the form of heterozygote advantage towards a large variety of pathogens following a divergent allele advantage (DAA) model, although negative frequencydependent (also named rare-allele advantage) and fluctuating selection in time and space also explain its remarkable variation [18-22]. These mechanisms maintain even $H L A$ allele frequencies in most populations, with recurrent - although not systematic - deviations from neutral expectations towards a significant excess of heterozygotes [21, 23]. However, specific HLA alleles may also act as protective factors to highly prevalent diseases and be selected positively, one of the best examples being the putative increase of $B * 53$ (B*53:01:01) and $B * 78$ ( $B * 78: 01)$ frequencies in sub-Saharan African regions where Plasmodium falciparum malaria is endemic [24-26]. Recently, $M H C$ alleles encoding for allotypes with functional similarities to those of $H L A-B=53$ and $H L A-\mathrm{B} * 78$ have also been suggested to play a protective role explaining the likely absence of malaria parasites in bonobos [27]. In addition, demographic processes such as population bottlenecks, genetic drift, demographic expansions or migrations shape the HLA molecular profiles by increasing or decreasing their diversity and create population structure most often highly correlated to geography [21, 28-30].

Whether and how $M H C$ genetic variation persists in populations having undergone a pronounced reduction in size, either due to a founder effect or to an epidemic, is an important issue in evolutionary genetics and conservation biology [30-34]. Indeed, a loss of genetic variation, particularly concerning immune-related loci, may have dramatic effects on populations' survival [33], even though a direct correlation between a lower $M H C$ diversity and a greater susceptibility to diseases has not been demonstrated so far at a population level [35, 36]. In this context, theoretical and empirical studies investigating the relative effects of genetic drift and natural selection on $M H C$ variability during population bottlenecks in different species have reported contrasting results, indicating either that balancing selection processes were efficient enough to maintain moderate to high $M H C$ diversity [31, 37-40] or that demographic factors exerted stronger influence than selection on diversity [41, 42]. Additionally, the impact of selection may depend both on the timescales, e.g. selection would be able to restore diversity to pre-bottleneck levels after 40 generations [31], and on the specific $M H C$ gene studied [38, 39, 41].

One useful approach to unravel the multiple mechanisms governing the evolution of the $M H C$ region is to compare the diversity of homologous genes among closely related species that underwent distinct demographic histories. This is the case for humans and chimpanzees, which share a common ancestor dating back to 6-8 million years (Myr) ago [43, 44]. According to both archaeological and genetic data, anatomically modern humans (Homo sapiens) first appeared and expanded demographically in Africa between 300,000 and 200,000 years ago $[45,46]$. They later dispersed, likely in small groups, across all continents where they eventually underwent secondary expansions, the most extensive ones (in Prehistoric times) occurring in the Neolithic $[47,48]$. However, many human populations (most Amerindian, Oceanian and present-day hunter-gatherer and nomadic populations from different continents) did not undergo demographic expansions [49] and still live today in isolated areas where they experience little gene flow and rapid genetic drift [50]. Due to the paucity of fossil records [51], the demographic history of chimpanzee populations relies almost exclusively on molecular analyses. The latter suggest the emergence of both common chimpanzees (Pan troglodytes, P.t. hereafter) and bonobos (Pan paniscus) in Central Africa from a common ancestor $\sim 1-2$ Myr ago [43, 44]; but while bonobos probably remained confined within the small geographic region where they inhabit today (a narrow territory between the Congo and Kasai Rivers), common chimpanzees expanded across a wider area of equatorial Africa where they are represented today by distinct subspecies (P.t.verus in Western Africa, P.t.ellioti in Nigeria and Cameroon, P.t.troglodytes in Central Africa, and P.t.schweinfurthii in Eastern Africa), albeit mainly within a limited rainforest habitat [52-54].

MHC molecular data analyses indicated that both common chimpanzees and bonobos experienced a selective sweep owing to the action of a hypothesised retroviral infection that severely shrunk their population sizes (bottleneck events) [55, 56]. The first evidence comes from the observation of a reduced repertoire of allele families at the Patr-A locus compared to the HLA$A$ locus in humans [57], suggesting a strong selective sweep - i.e. either purifying or positive directional selection - within the chimpanzees' $M H C$ class I region. Indeed, whereas $H L A-A$ alleles belong to six different allele families ( $A 2, A 10$ and $A 19$ within the $A 2$ lineage, and $A 1 / A 3 / A 11 / A 30, A 9$ and $A 80$ within the $A 3$ lineage), all Patr- $A$ alleles known so far are associated to the single $A 1 / A 3 / A 11 / A 30$ family [57-62] and a similar observation has been reported for the Papa-A alleles [63] (Papa is the name of $M H C$ genes in bonobo). Next, Patr- and 
Papa- $A,-B,-C$ intron 2 analyses substantiated the reduced diversity observed in the Western chimpanzee (P.t.verus) and bonobo $M H C$ class I regions as compared to $H L A-A,-B,-C$ in humans $[55,63,64]$. In addition, microsatellite analyses in Western chimpanzees and humans revealed a reduced diversity in the Patr region in comparison to microsatellites located elsewhere in the genome [56]. Finally, chimpanzees were shown to exhibit a $95 \mathrm{~kb}$ deletion in the $M I C$ region located next to locus $B$ where the single $M I C$ gene, which is fixed on all haplotypes, likely results from the fusion of two ancestral $M I C A$ and $M I C B$ genes still present in humans [65]. The hypothesis of a selective sweep proposed for chimpanzees finds support in the low genomic diversity found in all common chimpanzee sub-species and in bonobos, which was ascribed to a bottleneck in the ancestors of both species [44]. In addition, these genome-wide analyses also highlighted a second bottleneck occurring later ( 500,000 years ago) in Western and NigeriaCameroon chimpanzees only (although not quite as severe for the latest), which would partially explain why P.t.verus generally displays lower molecular variation in nuclear genes compared to other chimpanzee (sub-)species $[44,66-71]$.

In this study, our objective is to assess whether the genetic diversity at different Patr genes, estimated by means of three different indexes, allelic richness, expected heterozygosity and nucleotide diversity, is significantly reduced in present-day Western chimpanzee as a possible response to their past bottlenecks compared to that of their HLA orthologs in human populations. The detection of a substantially reduced level of Patr diversity would be a possible indicator of depleted immunity and an additional reason to consider P.t.verus as a critically endangered subspecies [72]. Actually, we anticipate chimpanzees' $M H C$ diversity to be (not necessarily similar but) closer to that of small isolated, as opposed to large outbred human populations (independently of their geographical location) if demographic contractions played a major role on the $M H C$ evolution of both species. In addition, we expect the patterns of genetic variation and linkage disequilibrium to be similar across the HLA and Patr regions if their orthologous loci evolved through analogous molecular mechanisms and were targeted by similar selective pressures in the two species. To address these issues, we analysed all the data currently available for 7 Patr genes $(A, B, C, D R B 1, D Q A 1$, $D Q B 1$ and $D P B 1$ ) in four P.t.verus cohorts, and we compared them to large sets of data for $H L A$ genes $(A, B, C$, $D R B 1, D Q A 1, D Q B 1$ and $D P B 1)$ data previously studied in human populations from different continents, that we also extensively reanalysed. We found marked similarities in Patr and HLA genetic diversity and linkage disequilibrium patterns, indicating highly conserved mechanisms of $M H C$ evolution in chimpanzees and humans. We also showed that Western chimpanzees globally exhibit similar diversity levels and equivalent amounts of linkage disequilibrium to those estimated in small isolated human populations, which suggests that their past bottleneck exerted a substantial effect on the molecular diversity of Patr genes. However, as there was no difference in the $\mathrm{MHC}$ diversity of chimpanzees compared to human populations that likely underwent more recent, rapid genetic drift, we hypothesize that several Patr genes rapidly recovered molecular variation after their selective sweep.

\section{Results}

\section{Hardy-Weinberg equilibrium and selective neutrality}

The results of Hardy-Weinberg equilibrium (HWE) and Ewens-Watterson-Slatkin (EWS) tests are provided in Table 1 (for the pooled chimpanzee cohort and the multiple human populations) and Additional Tables S1 (for the individual chimpanzee cohorts) and S2 (for the individual human populations).

No deviation from HWE was observed at any Patr locus for any of the four individual cohorts and the pooled cohort of chimpanzees. The computed allele frequencies (see below) could thus accurately be used as population frequencies to compare cohorts among them and with human populations as well as to estimate other parameters requiring HWE (e.g. heterozygosity). Additionally, we found no significant deviations (after correction for multiple testing) of allele frequency distributions from neutral expectations based on the EWS test.

All human populations were also found to be in HWE both before (except the Mixe (Mexico/Oaxaca) at DRB1) and after correction for multiple testing. Contrary to chimpanzees, however, a few significant rejections of selective neutrality were still found in human populations after correction for multiple testing, i.e. towards an excess of heterozygotes at loci $A(3.7 \%), D R B 1$ (7.9\%) and $D Q B 1(2.5 \%)$ and towards an excess of homozygotes at locus $D P B 1$ (2\%), but none at loci $D Q A 1, B$ and $C$.

To control for the large differences in sample sizes between chimpanzees (average $N=45.57 \pm 7.76$ on the 7 loci in the pooled cohort) and humans (average $N=$ $109.2 \pm 17.31$ on the 7 loci and the multiple populations), we also tested HWE and selective neutrality on 1000 simulated sub-samples drawn randomly from each human population, each simulated sub-sample being of same size as the pooled cohort of chimpanzees (see Methods). As a result, we observed various proportions of HWE deviations in the simulated sub-samples depending on the locus (average proportion $\pm 2 \mathrm{x}$ Standard Error, $D P B 1: \quad 8.05 \% \pm 8.20 \%, \quad D Q B 1: \quad 13.38 \pm 9.83 \%$, $D Q A 1: \quad 6.89 \pm 9.40 \%, \quad D R B 1: \quad 10.37 \pm 8.99 \%, \quad B: 3.36 \pm$ 
Table 1 Results of Hardy-Weinberg equilibrium (HWE) and Ewens-Watterson-Slatkin (EWS) tests at seven MHC loci in chimpanzees (pooled cohort) and humans (multiple populations)

\begin{tabular}{|c|c|c|c|c|c|c|c|}
\hline Chimpanzees (pooled cohort) & DPB1 & $D Q B 1$ & $D Q A 1$ & $D R B 1$ & B & C & $A$ \\
\hline $\bar{N}$ & 44 & 48 & 29 & 46 & 51 & 51 & 50 \\
\hline HWE $p$-value & 1 & 0.456 & 0.627 & 0.81 & 0.983 & 0.226 & 1 \\
\hline EWS p-value (excess heterozygotes) & 0.079 & 0.873 & 0.100 & 0.031 & 0.645 & 0.811 & 0.069 \\
\hline EWS p-value (excess homozygotes) & 0.491 & 0.970 & 0.707 & 0.473 & 0.907 & 0.945 & 0.572 \\
\hline Humans (multiple populations) & DPB1 & DQB1 & $D Q A 1$ & $D R B 1$ & B & C & $A$ \\
\hline k & 50 & 79 & 52 & 89 & 80 & 59 & 81 \\
\hline $\bar{N}(s . d)$ & $87.8(45.2)$ & $100.6(55.3)$ & $90.7(45.5)$ & $105.5(111.8)$ & $124(134.6)$ & $127.9(141.1)$ & $127.6(133.6)$ \\
\hline \% HWE rejections (after correction) & 0 & 0 & 0 & $1.1(0)$ & 0 & 0 & 0 \\
\hline \% EWS rejections (excess heterozygotes) & 0 & $16.46(2.5)$ & $28.84(0)$ & $30.34(7.9)$ & $30(0)$ & $33.9(0)$ & $16.05(3.7)$ \\
\hline \% EWS rejections (excess homozygotes) & $22(2)$ & $1.27(0)$ & 0 & $2.25(0)$ & $5(0)$ & $1.69(0)$ & $7.41(0)$ \\
\hline
\end{tabular}

$\mathrm{N}$ : sample size (in number of individuals); k: number of populations; $\bar{N}$ : mean sample size (in number of individuals); s.d.: standard deviation; Holm correction for multiple testing is given within brackets for the \% of HWE and EWS tests. Significance tests were done without prior assumptions, thus two-tailed rejection at the $5 \%$ level either occurs below 0.025 (excess of heterozygotes) or above 0.975 (excess of homozygotes). The order of loci corresponds to their position on the chromosome from centromere (left) to telomere (right). The pooled cohort does not include Texas ${ }^{\mathrm{cb}}$ (see Text)

$5.05 \%, C: 3.20 \pm 4.93 \%, A: 5.77 \pm 6.6 \%$, Additional Table S2). As almost all human populations of the original dataset were in HWE, this overall result allowed us to conclude that a reduction in sample size sometimes leads to type I errors, i.e. false positives, at loci $D Q B 1$ and $D R B 1$ (the only proportions significantly different from 0). However, for the Mixe from Mexico/Oaxaca, which was the only population for which HWE was rejected before correction for multiple testing (at locus $D R B 1)$ in the original dataset, HWE was rejected in all (i.e. the 1000) simulated sub-samples, a result that never occurred otherwise (Additional Table S2). This indicates that the power of the test strongly resists a reduction in sample size, and that the observation of no HWE rejection in the chimpanzee samples truly reflects HWE in the corresponding cohorts.

Regarding selective neutrality, our simulations failed to reject the null hypothesis in various proportions of simulated sub-samples drawn from populations for which neutrality was initially rejected (10\% at locus DPB1, $2.1 \%$ at locus $D Q B 1,28.3 \%$ at locus $D R B 1$ and $18.4 \%$ at locus $A$, Additional Table $\mathrm{S} 2$ ). In this case, the absence of significant deviations from neutrality observed in chimpanzees could thus correspond to type II errors, i.e. false negatives, due to a lack of power of the neutrality test when applied to small sample sizes, although this occurred in a minority of cases according to our simulations (less than 30\%).

\section{Genetic diversity}

Allele frequencies estimated in the pooled cohort of chimpanzees are given in Table 2 and Additional Table $\mathrm{S} 1$ (for the individual chimpanzee cohorts). Allelic distributions found at the three class I loci $B, C$ and $A$ and at $D R B 1$ are much more diverse than those observed at
$D Q B 1$ and $D Q A 1$ and, to a lesser extent, $D P B 1$. Moreover, at loci $D Q B 1$ and $D Q A 1$, three alleles account for more than $84.5 \%$ of frequencies. A greater number of low frequency alleles are observed for loci $B, C$ and $A$ than for class II loci (in light grey in Table 2, see also SupplementaryText for a comparison between chimpanzee cohorts and human populations).

The three genetic diversity indexes estimated at the seven Patr genes in the four cohorts and the pooled cohort of chimpanzees are given in Table 3 and plotted in Fig. 2, and the corresponding values are provided in Additional Table S3.

In agreement with the observed allele frequency distributions, both allelic richness and heterozygosity show greater values at the three class I loci $A, B, C$ and at $D R B 1$ than at $D Q A 1, D Q B 1$ and $D P B 1$ (to a lesser extent for the latter). Based on the loci for which data were available in (at least one) captive and wild cohorts $(D Q B 1, D R B 1, B, C, A)$, we also observe significantly higher values of these indexes in the captive-born Tex$\mathrm{as}^{\mathrm{cb}}$ and Yerkes ${ }^{\mathrm{cb}}$ than in the wild-born BPRC ${ }^{\mathrm{wb}}$ and Kuma $^{\text {wb }}$ cohorts (Wilcoxon tests, $p=0.0036$ and $p=$ 0.0034 , for allelic richness and heterozygosity, respectively). By contrast, nucleotide diversity is greater at $D R B 1, D Q A 1, D Q B 1$ and $B$ (to a lesser extent in the two latter) than at $A, C$ and $D P B 1$, and no significant differences are observed between the cohorts (Wilcoxon test, $p=0.769)$.

Like in chimpanzees, both the allelic richness and the heterozygosity estimated in human populations are, on average, greater at the three class I loci $A, B, C$ and at $D R B 1$ than at $D Q A 1, D Q B 1$ and $D P B 1$ and the nucleotide diversity is greater at loci $D R B 1, D Q A 1, D Q B 1$ and $B$ than at $A, C$ and $D P B 1$ (Table 3 and Fig. 2). The overall patterns of genetic diversity are therefore similar in 
Table 2 Allele frequencies at each Patr locus in the pooled cohort of chimpanzees ${ }^{a}$

\begin{tabular}{|c|c|c|c|c|c|c|c|c|c|c|c|c|c|}
\hline \multicolumn{2}{|l|}{ Locus DPB1 } & \multicolumn{2}{|l|}{ Locus DQB1 } & \multicolumn{2}{|l|}{ Locus DQA $7^{\mathrm{b}}$} & \multicolumn{2}{|l|}{ Locus DRB1 } & \multicolumn{2}{|l|}{ Locus B } & \multicolumn{2}{|l|}{ Locus C } & \multicolumn{2}{|l|}{ Locus A } \\
\hline$N=44$ & & $N=48$ & & $N=29$ & & $N=46$ & & $N=51$ & & $N=51$ & & $N=50$ & \\
\hline DPB1*01:09 & 0.279 & $\mathrm{DQB} 1 * 03: 02$ & 0.5937 & DQA1*20:04 & 0.4138 & DRB1*02:01 & 0.2283 & $B^{*} 01: 01$ & 0.2807 & $C^{*} 04: 01$ & 0.3075 & $A^{*} 03: 01$ & 0.1505 \\
\hline DPB1*01:11 & 0.2514 & DQB1*06:02 & 0.2917 & DQA1*01:01 & 0.2759 & DRB1*02:04 & 0.2283 & $B^{*} 05: 01$ & 0.1764 & $C^{*} 06: 01$ & 0.2629 & $A^{*} 04: 01$ & 0.126 \\
\hline $\mathrm{DPB}$ *01:12 & 0.1705 & $\mathrm{DQB} 1 * 06: 01$ & 0.0521 & DQA $1 * 05: 02$ & 0.1552 & DRB1*03:07 & 0.1528 & $B^{*} 13: 01$ & 0.0784 & $C^{*} 09: 01$ & 0.1211 & $A^{*} 09: 01$ & 0.126 \\
\hline DPB1*01:07 & 0.1073 & DQB1*15:01 & 0.0312 & DQA1*20:01 & 0.1034 & DRB1*03:02 & 0.0913 & $B^{*} 24: 02$ & 0.0783 & $C^{*} 02: 03$ & 0.0686 & $A^{*} 07: 01$ & 0.1193 \\
\hline $\mathrm{DPB} 1 * 01: 13$ & 0.1023 & DQB1*03:05 & 0.0208 & $\mathrm{DQA} 1 * 05: 03$ & 0.0517 & DRB1*03:05 & 0.087 & $B^{*} 17: 01$ & 0.0686 & $C^{*} 11: 01$ & 0.049 & $A^{*} 01: 01$ & 0.0983 \\
\hline DPB1*01:18 & 0.0114 & DQB1*06:07 & 0.0104 & & & DRB1*03:09 & 0.0462 & $B^{*} 14: 01$ & 0.0588 & $C^{*} 05: 01$ & 0.0392 & $A^{*} 14: 01$ & 0.07 \\
\hline DPB1*03:04 & 0.0114 & & & & & DRB1*10:01 & 0.0435 & $B^{*} 24: 01$ & 0.0392 & $C^{*} 12: 01$ & 0.033 & $A^{*} 06: 01$ & 0.0668 \\
\hline \multirow[t]{14}{*}{ blank } & 0.0668 & & & & & DRB1*03:11 & 0.0326 & $B^{*} 04: 01$ & 0.0294 & $C^{*} 03: 01$ & 0.0196 & $A^{*} 05: 01$ & 0.0422 \\
\hline & & & & & & DRB1*07:01 & 0.0217 & $B^{*} 16: 01$ & 0.0294 & $C^{*} 05: 02$ & 0.0196 & $A^{*} 02: 01$ & 0.0319 \\
\hline & & & & & & blank & 0.0684 & $B^{*} 20: 01$ & 0.0294 & $C^{*} 01: 01$ & 0.0114 & $A^{* 11: 01}$ & 0.03 \\
\hline & & & & & & & & $B^{*} 03: 01$ & 0.0214 & $C^{*} 02: 02$ & 0.0098 & $A^{*} 06: 02$ & 0.0106 \\
\hline & & & & & & & & $\mathrm{B}^{*} 09: 01$ & 0.0214 & $C^{*} 08: 01$ & 0.0098 & $A^{*} 03: 02$ & 0.01 \\
\hline & & & & & & & & $B^{*} 29: 01$ & 0.0196 & $C^{*} 09: 02$ & 0.0098 & $A^{*} 04: 02$ & 0.01 \\
\hline & & & & & & & & $B^{*} 05: 02$ & 0.0098 & $C^{*} 13: 02$ & 0.0098 & $A^{*} 04: 04$ & 0.01 \\
\hline & & & & & & & & $B^{*} 08: 02$ & 0.0098 & blank & 0.0288 & $A^{*} 08: 02$ & 0.01 \\
\hline & & & & & & & & $B^{*} 10: 01$ & 0.0098 & & & $A^{*} 08: 03$ & 0.01 \\
\hline & & & & & & & & $B^{*} 16: 02$ & 0.0098 & & & $A^{*} 17: 01$ & 0.01 \\
\hline & & & & & & & & $B^{*} 19: 01$ & 0.0098 & & & blank & 0.0683 \\
\hline & & & & & & & & $B^{*} 22: 01$ & 0.0098 & & & & \\
\hline & & & & & & & & $B^{*} 23: 01$ & 0.0098 & & & & \\
\hline & & & & & & & & blank & 0.0002 & & & & \\
\hline
\end{tabular}

Alleles in grey have a frequency lower than 0.05

${ }^{a}$ The allele frequencies for the individual chimpanzee cohorts are in Additional Table S1

${ }^{\mathrm{b}}$ For locus $D Q A 1$, only data for cohort $\mathrm{BPRC}^{\mathrm{wb}}$ are available

the two species. This is also supported by comparing the ordering of the seven $M H C$ loci based on decreasing values of the three diversity indexes (Table 4): identical orders are found for several loci, and small differences are most often observed otherwise. These results suggest that the mechanisms generating diversity at the $M H C$ genes are similar, and thus highly conserved, in the human and chimpanzee lineages.

Looking in more detail at the results obtained for individual $M H C$ genes, some significant differences are nevertheless observed between the two species. Compared to humans, in chimpanzees we find a lower heterozygosity, allelic richness and nucleotide diversity at DQB1 (Wilcoxon test, $p=0.016,0.017$ and 0.021 , respectively), as well as a lower nucleotide diversity at $C$ and $A$ (Wilcoxon test, $p=0.011$ and 0.019 , respectively) and a higher nucleotide diversity at $B$ (Wilcoxon test, $p=0.009$ ) (Table 3 and Fig. 2). We obtained similar results by redoing these comparisons without considering the Texas ${ }^{\mathrm{cb}}$ cohort, which includes individuals of uncertain sub-species (Wilcoxon test: $p=0.019,0.03$ and 0.041 for heterozygosity, allelic richness and nucleotide diversity at DQB1; and $p=0.025,0.013$ and 0.047 for nucleotide diversity at $B, C$, and $A$, respectively, see also Additional Figure S1). However, according to both sets of comparisons (i.e. with and without the Texas ${ }^{\mathrm{cb}}$ cohort), none of these differences remained significant after correction for multiple testing on the number of loci. This confirmed our previous conclusion that chimpanzees and humans display similar patterns of genetic diversity across the whole $M H C$ region (Fig. 2, left and central panes).

\section{Genetic diversity in chimpanzees compared to small and large human populations}

Following the idea, based on demographic knowledge, that chimpanzees would be genetically more similar to human populations displaying limited population sizes, we also compared the three diversity indexes between the chimpanzees and the human populations classified either as $R G D$ (small isolated populations that likely underwent Rapid Genetic Drift) or as SGD (large outbred populations those that likely underwent Slow Genetic Drift), respectively (see Methods).

Interestingly, in the chimpanzee cohorts - and particularly so in the wild-born BPRC ${ }^{\mathrm{wb}}$ and $\mathrm{Kuma}^{\mathrm{wb}}$ - both the 
Table 3 Genetic diversity at 7 MHC loci in chimpanzees (average on all chimpanzee cohorts and in the pooled cohort) and human populations (averaged on multiple populations)

\begin{tabular}{|c|c|c|c|c|c|c|c|c|c|c|c|c|c|}
\hline & \multicolumn{4}{|c|}{ Number of samples $(k) \&$ sample size $(N)$} & \multicolumn{3}{|c|}{ Allelic richness (ar) } & \multicolumn{3}{|c|}{ Heterozygosity $(H)$} & \multicolumn{3}{|c|}{ Nucleotide diversity $(\Pi)$} \\
\hline & \multicolumn{2}{|c|}{ Chimpanzees } & \multicolumn{2}{|c|}{ Humans } & \multicolumn{2}{|c|}{ Chimpanzees } & \multirow{3}{*}{$\begin{array}{l}\text { Humans } \\
\overline{a r}^{p} \text { (s.d) }\end{array}$} & \multicolumn{2}{|c|}{ Chimpanzees } & \multirow{3}{*}{$\begin{array}{l}\text { Humans } \\
\bar{H} \% \text { (s.d) }\end{array}$} & \multicolumn{2}{|c|}{ Chimpanzees } & \multirow{3}{*}{$\begin{array}{l}\text { Humans } \\
\bar{\Pi} \times 10^{-2} \\
\text { (s.d) }\end{array}$} \\
\hline & & & & & \multirow{2}{*}{$\begin{array}{l}\text { average } \\
\overline{\operatorname{ar}}(s . d)\end{array}$} & \multirow{2}{*}{$\begin{array}{l}\text { pooled }^{a} \\
\operatorname{ar}^{p}\end{array}$} & & \multirow{2}{*}{$\begin{array}{l}\text { average } \\
\bar{H} \% \text { (s.d) }\end{array}$} & \multirow{2}{*}{$\begin{array}{l}\text { pooled }^{a} \\
H^{P} \%\end{array}$} & & \multirow{2}{*}{$\begin{array}{l}\text { average } \\
\bar{\Pi} \times 10^{-2} \\
\text { (s.d) }\end{array}$} & \multirow{2}{*}{$\begin{array}{l}\text { pooled }^{a} \\
\Pi^{p} \times \\
10^{-2}\end{array}$} & \\
\hline & k & $N^{P}$ & k & $\bar{N}(s . d)$ & & & & & & & & & \\
\hline$D P B 1$ & 2 & 44 & 50 & $87.8(45.2)$ & $6.3(1.8)$ & 7.4 & $9.8(3.7)$ & $77.9(0.4)$ & 80.3 & $\begin{array}{l}72.5 \\
(17.1)\end{array}$ & $1.3(0.1)$ & 1.3 & $2.4(0.9)$ \\
\hline DQB1 & 3 & 48 & 79 & $100.6(55.3)$ & $\begin{array}{l}5.0^{*} \\
(0.9)\end{array}$ & 5.6 & $9.6(3.2)$ & $\begin{array}{l}63.7^{*} \\
(13.8)\end{array}$ & 55.8 & 79 (11.2) & $4.0^{*}(0.3)$ & 3.9 & $6.0(1.6)$ \\
\hline DQA1 & 1 & 29 & 52 & $90.6(45.5)$ & $5(-)$ & 5 & $6.4(1.6)$ & $71.5(-)$ & 71.5 & $\begin{array}{l}74.3 \\
(10.9)\end{array}$ & $7.8(-)$ & 7.8 & $6.9(1.3)$ \\
\hline DRB1 & 3 & 46 & 89 & 105.5 (111.8) & $\begin{array}{l}10.1 \\
(2.6)\end{array}$ & 9.7 & $\begin{array}{l}14.3 \\
(5.3)\end{array}$ & $84.7(3.8)$ & 84.6 & $86.4(8.3)$ & $8.1(1.1)$ & 7.0 & $7.0(1.2)$ \\
\hline B & 3 & 51 & 80 & $124(134.6)$ & $\begin{array}{l}13.9 \\
(3.5)\end{array}$ & 17.1 & $\begin{array}{l}19.2 \\
(7.1)\end{array}$ & 84.1 (3.9) & 86.4 & $90.3(6.8)$ & $\mathbf{5 . 0} *(0.5)$ & 5.2 & $4.2(0.5)$ \\
\hline C & 2 & 51 & 59 & $127.9(141.1)$ & $9.7(3.2)$ & 11.5 & $\begin{array}{l}12.4 \\
(4.2)\end{array}$ & 79.6 (4.3) & 81 & $85.2(7.4)$ & $\mathbf{2 . 1} *(0.2)$ & 2.1 & $2.6(0.2)$ \\
\hline$A$ & 3 & 50 & 81 & 127.6 (133.6) & $13(0.9)$ & 14.2 & $\begin{array}{l}11.9 \\
(5.2)\end{array}$ & $88.6(1.2)$ & 90.3 & $\begin{array}{l}79.7 \\
(15.4)\end{array}$ & $\mathbf{2 . 7} *(0.3)$ & 2.7 & $3.4(0.5)$ \\
\hline
\end{tabular}

$k$ : number of samples; $N^{p}$ : size of pooled cohort; $\bar{N}(s . d)$ : average size of human population samples and standard deviation

$\overline{a r}(s . d)$ : average allelic richness in the chimpanzee samples and standard deviation; $\operatorname{ar}^{P}$ : allelic richness of the pooled cohort; $\overline{\operatorname{ar}}^{P}$ (s.d): average allelic richness in human population samples (estimated relatively to the pooled cohort) and standard deviation. ${ }^{*}$ value (in bold) significantly different between human populations

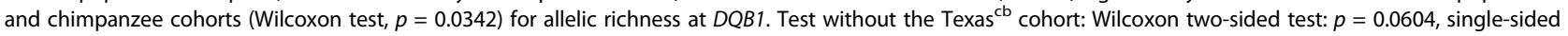
test ("less"): $p=0.0302$

$\bar{H} \%$ (s.d): average expected heterozygosity in the chimpanzee samples and in human population samples and standard deviation; $H^{P} \%$ : expected heterozygosity of the pooled cohort. * value (in bold) significantly different between human populations and chimpanzee cohorts (Wilcoxon test, $p=0.0316$ ) for heterozygosity at DQB1. Test without the Texas ${ }^{\mathrm{cb}}$ cohort: Wilcoxon two-sided test: $p=0.0371$, single-sided test ("less"): $p=0.0185$

$\bar{\Pi}\left(\right.$ s.d): average expected nucleotide diversity in the chimpanzee samples and in human population samples and standard deviation; $\Pi^{P}$ : expected nucleotide diversity of the pooled cohort. * values (in bold) significantly different between human populations and chimpanzee cohorts (Wilcoxon test, $p=0.0426,0.0175$,

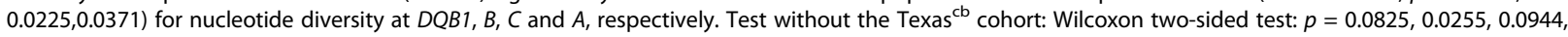
single-sided test: $p=0.0412,0.0128,0.0472$ at $D Q B 1, B$ and $A$, respectively

The order of loci corresponds to their position on the chromosome from centromere (top) to telomere (bottom)

${ }^{\mathrm{a}}$ does not include Texas ${ }^{\mathrm{cb}}$ (see Text)

allelic richness and heterozygosity (at all loci except $A$ ) are close to the lowest values found for these indexes in human populations, which correspond to those observed in $R G D$ populations (Fig. 2, right graphs). Actually, at these loci, chimpanzees exhibit no significant differences compared to $R G D$ populations, whereas all differences (except heterozygosity at DPB1) are significant compared to $S G D$ populations. In addition, chimpanzees exhibit significant nucleotide diversity differences compared to $S G D$ populations at three loci, DPB1, $D Q B 1$ and $A$ (Additional Table S4). After correction for the number of loci tested, the three diversity indexes appear to be both similar between chimpanzees and $R G D$ populations at all loci (except one borderline case, nucleotide diversity at locus $B$ ) and different between chimpanzees and $S G D$ populations (at least two loci remain highly significant after correction). This strongly suggests that demographic contractions globally exerted a similar effect - i.e. a decrease in the level of diversity on Patr and HLA genes.

Again to control for the discrepancy in sample sizes between chimpanzees and humans, we re-estimated allelic richness, heterozygosity and nucleotide diversity on 1000 simulated sub-samples randomly drawn for each human population. For the three diversity indexes, the values (in all cases at a precision of one decimal, but most often, even at two) observed for the original human population samples were always found to fall within the 95\% confidence interval of their simulated subsamples (Additional Table S2). In addition, the relative position of each genetic diversity index observed in the pooled cohort of chimpanzees - i.e. either within or outside the $95 \%$ confidence interval - was identical when compared both to the confidence interval of the original human population samples and to that of the 1000 simulated sub-samples (Additional Figure S2). This substantiated our previous conclusion that chimpanzees and human $R G D$ populations exhibit similar $M H C$ diversity patterns.

\section{Linkage disequilibrium}

In chimpanzees, global linkage disequilibrium (GLD) appears to be significant between the three class II loci $D Q A 1, D Q B 1$ and $D R B 1$ (i.e. pairs $D Q A 1 \sim D R B 1$, 


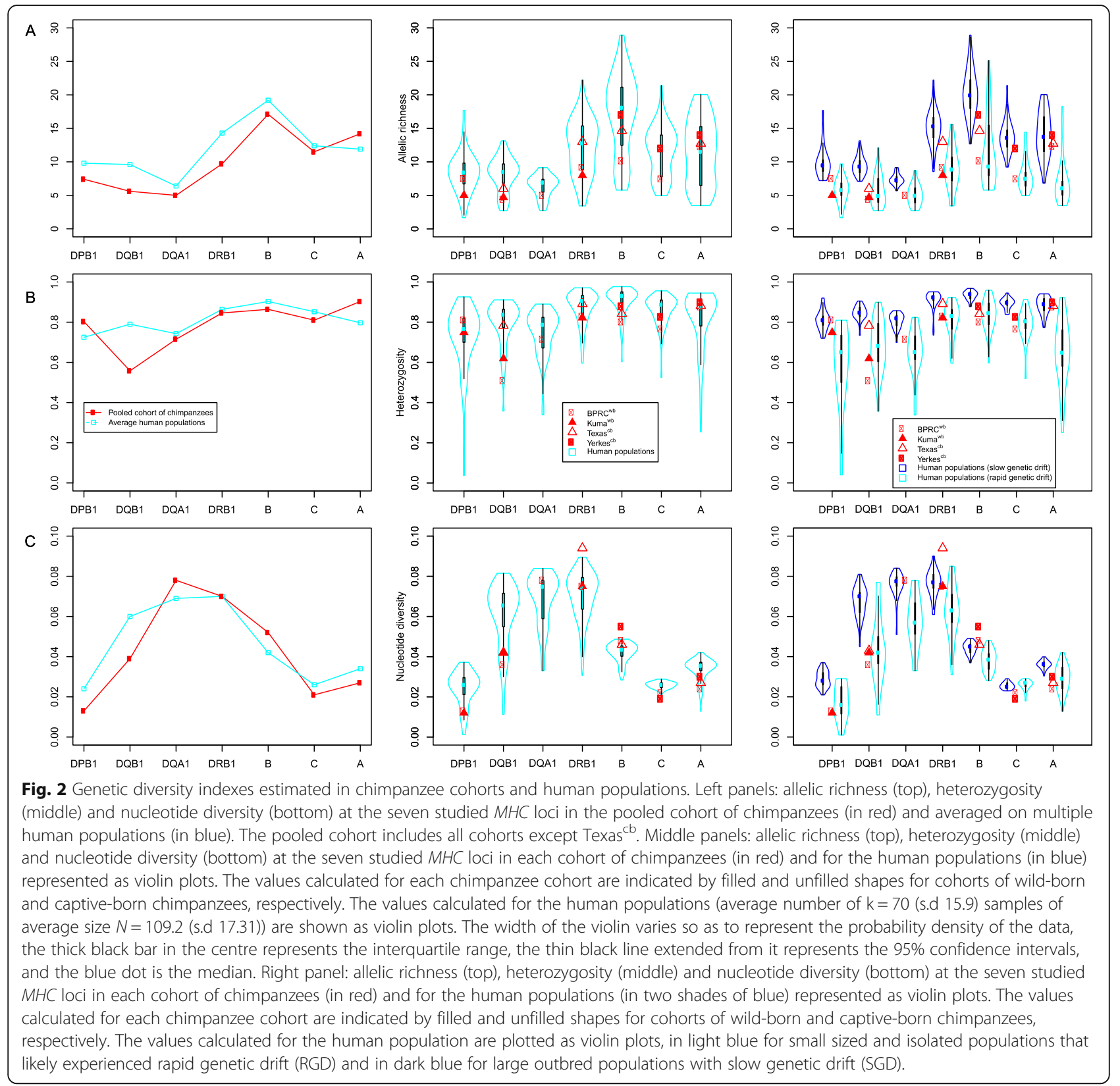

Table 4 Ordering of the MHC loci based on decreasing values of three genetic diversity indexes in chimpanzees (pooled cohort) and humans (average on multiple populations)

\begin{tabular}{|c|c|c|}
\hline Genetic diversity & Species & Ordering of values \\
\hline \multirow[t]{2}{*}{ Allelic richness } & Chimpanzees (pooled cohort ${ }^{\&}$ ) & $B>A>C>D R B 1>D P B 1>D Q B 1>D Q A 1$ \\
\hline & Humans (average & $B>D R B 1>C>A>D P B 1>D Q B 1>D Q A 1$ \\
\hline \multirow[t]{2}{*}{ Heterozygosity } & Chimpanzees (pooled cohort ${ }^{\&}$ ) & $A>B>D R B 1>C>D P B 1>D Q A 1>D Q B 1$ \\
\hline & Humans (average") & $B>D R B 1>C>A>D Q B 1>D Q A 1>D P B 1$ \\
\hline \multirow[t]{2}{*}{ Nucleotide diversity } & Chimpanzees (pooled cohort ${ }^{\&}$ ) & $D Q A 1>D R B 1>B>D Q B 1>A>C>D P B 1$ \\
\hline & Humans (average ${ }^{\#}$ ) & $D R B 1>D Q A 1>D Q B 1>B>A>C>D P B 1$ \\
\hline
\end{tabular}

Perfect matches in locus ordering between chimpanzees and humans are highlighted in bold; ${ }^{*}$ estimated on multiple human populations; ${ }^{\&}$ for chimpanzees, the pooled cohort has an average sample size of 45.57 on the different loci; ${ }^{\#}$ : for humans, the averages were calculated on an average number of 70.14 populations with an average sample size of 109.46 . The pooled cohort does not include Texas ${ }^{\mathrm{cb}}$ (see Text) 
Table 5 Results of Global Linkage Disequilibrium (GLD) significance test (PRS resampling procedure) between different pairs of MHC loci in chimpanzees (BPRC cohort) and humans (multiple populations, further subdivided into RGD and SGD populations)

\begin{tabular}{|c|c|c|c|c|c|c|c|c|}
\hline \multirow[b]{3}{*}{ Loci pairs } & \multirow{2}{*}{\multicolumn{2}{|c|}{$\frac{\text { Chimpanzees }}{\text { BPRC }^{\text {WB }}}$}} & \multicolumn{6}{|c|}{ Humans } \\
\hline & & & \multicolumn{2}{|c|}{ Multiple populations } & \multicolumn{2}{|c|}{ RGD } & \multicolumn{2}{|c|}{ SGD } \\
\hline & $N$ & $p$ & k & $\begin{array}{l}\% \text { significant results } \\
\text { (after correction) }\end{array}$ & $k 1$ & $\begin{array}{l}\text { \% significant results } \\
\text { (after correction) }\end{array}$ & $k 2$ & $\begin{array}{l}\% \text { significant results } \\
\text { (after correction) }\end{array}$ \\
\hline$D P B 1 \sim D Q B 1$ & 25 & 0.106 & 40 & $30(12.5)$ & 15 & $47(27)$ & 25 & $24(4)$ \\
\hline$D P B 1 \sim D Q A 1$ & 25 & 0.0697 & 33 & $27.3(15.1)$ & 16 & $44(25)$ & 17 & $12(5.9)$ \\
\hline$D P B 1 \sim D R B 1$ & 25 & 0.328 & 31 & $25.8(9.7)$ & 16 & $37(12.5)$ & 15 & $13(6.7)$ \\
\hline$D P B 1 \sim B$ & 25 & 1 & 10 & $10(10)$ & 4 & $25(25)$ & 6 & 0 \\
\hline$D P B 1 \sim C$ & 25 & 0.999 & 8 & $12.5(0)$ & 2 & $50(0)$ & 6 & 0 \\
\hline$D P B 1 \sim A$ & 24 & 0.982 & 12 & 0 & 5 & 0 & 7 & 0 \\
\hline$D Q B 1 \sim D Q A 1$ & 29 & $<10^{-4}$ & 46 & $97.8(97.8)$ & 20 & $95(95)$ & 26 & $100(100)$ \\
\hline$D Q B 1 \sim D R B 1$ & 29 & $<10^{-4}$ & 51 & $94.1(94.1)$ & 23 & $96(96)$ & 28 & $93(93)$ \\
\hline$D Q B 1 \sim B$ & 29 & 1 & 13 & $61.5(53.8)$ & 7 & $86(86)$ & 6 & $33(16.7)$ \\
\hline$D Q B 1 \sim C$ & 29 & 0.975 & 10 & $40(40)$ & 5 & $60(60)$ & 5 & $20(20)$ \\
\hline$D Q B 1 \sim A$ & 28 & 0.98 & 16 & $12.5(12.5)$ & 9 & $22(22)$ & 7 & 0 \\
\hline$D Q A 1 \sim D R B 1$ & 29 & $<10^{-4}$ & 38 & $97.4(97.4)$ & 21 & $100(100)$ & 17 & $94(94)$ \\
\hline$D Q A 1 \sim B$ & 29 & 1 & 9 & $44.4(44.4)$ & 5 & $80(80)$ & 4 & 0 \\
\hline$D Q A 1 \sim C$ & 29 & 1 & 6 & $33.3(33.3)$ & 3 & $67(67)$ & 3 & 0 \\
\hline$D Q A 1 \sim A$ & 28 & 0.831 & 12 & $8.3(0)$ & 7 & $14(0)$ & 5 & 0 \\
\hline$D R B 1 \sim B$ & 29 & 1 & 39 & $51.3(35.9)$ & 22 & $73(54.5)$ & 17 & $23(11.8)$ \\
\hline$D R B 1 \sim C$ & 29 & 0.998 & 30 & $63.3(63.3)$ & 18 & $78(78)$ & 12 & $42(42)$ \\
\hline$D R B 1 \sim A$ & 28 & 0.991 & 39 & $28.2(25.6)$ & 24 & $33(33)$ & 15 & $20(13.3)$ \\
\hline$B \sim C$ & 29 & 0.0001 & 59 & $74.6(74.6)$ & 21 & $95(95)$ & 38 & $63(63)$ \\
\hline$B \sim A$ & 28 & 0.931 & 76 & $35.5(25)$ & 27 & $63(40.7)$ & 49 & $20(16.3)$ \\
\hline$C \sim A$ & 28 & 0.892 & 58 & $53.4(37.9)$ & 23 & $65(47.8)$ & 35 & $46(31.4)$ \\
\hline
\end{tabular}

The PRS resampling procedure was done with $10^{\prime} 000$ simulations for the chimpanzee cohorts (significant results are in bold). The PRS resampling procedure was done with 1'000 simulations in each human population, and the table reports the percentage of significant results for each pair of loci (in brackets after Holm correction for multiple testing). Pairs in significant global linkage disequilibrium in more than $70 \%$ of human populations are in bold. $N$ : number of chimpanzee individuals; $p$ : $p$-value; $k$ : total number of human populations; $k 1$ : number of $R G D$ populations; $k 2$ : number of SGD populations. The table includes results obtained for the BPRC ${ }^{\text {wb }}$ cohort, i.e. the cohort including the greatest number of animals and the only one for which all loci were tested. Results for the other cohorts are in Additional Table S5

$D Q B 1 \sim D R B 1$ and $D Q B 1 \sim D Q A 1)$ as well as between the two class I loci $B$ and $C$ (pair $B \sim C$ ) (Table 5, see also SupplementaryText), as indicated by the results obtained for the BPRC ${ }^{\mathrm{wb}}$ cohort, i.e. the cohort including the greatest number of animals and the only one for which all loci were tested (Additional Table S5). These pairs of loci (actually those that are most close to each other on the chromosome, see Fig. 1) also display the highest proportions of individual haplotypes in linkage disequilibrium (Additional Tables S6 and S7), which strongly supports the observed GLD pattern.

These results are again similar in humans. Indeed, significant GLD is observed for the same pairs of loci $D Q A 1 \sim D R B 1, D Q B 1 \sim D R B 1, D Q B 1 \sim D Q A 1$ and $B \sim C$ in the majority (more than $70 \%$ and up to $98 \%$ ) of human populations (Table 5), and the highest proportions of individual haplotypes in significant linkage disequilibrium are also observed at these loci pairs in humans (Additional Table S6 and Additional Table S8, respectively). Therefore, as for genetic diversity, the patterns of linkage disequilibrium observed across the $M H C$ loci are highly conserved in the human and chimpanzee lineages.

\section{Linkage disequilibrium in chimpanzees compared to small and large human populations}

When comparing human $R G D$ and $S G D$ populations, the highest proportion of significant GLD are always found among the former, except for one pair of loci, $D Q B 1 \sim D Q A 1$ (Table 5). Actually, we find both significantly higher proportions of GLD and significantly higher average proportions of haplotypes in linkage disequilibrium in $R G D$ than in $S G D$ populations (Wilcoxon test: $p=0.014$ and $p=0.012$ ) (Additional Table S6 and Additional Table S8), which indicates that, globally, 
demography (i.e. genetic drift) did play a substantial role in the generation of linkage disequilibrium at the $H L A$ loci. However, this effect appears to be less pronounced at the $D Q A 1 \sim D R B 1, D Q B 1 \sim D R B 1, D Q B 1 \sim D Q A 1$ pairs.

Simulations performed on 1000 randomly drawn human population sub-samples show a tendency to underestimate GLD when sample sizes are low except for pairs $D Q B 1 \sim D Q A 1, D Q B 1 \sim D R B 1, D Q A 1 \sim D R B 1$ and $B \sim C$ (considering samples with GLD in more than 900 subsamples, we observe between half to two thirds less GLD in the simulated sub-samples except at these four loci pairs) (Additional Table S9). This suggests that the nondetection of significant GLD in chimpanzees for other loci than $D Q A 1 \sim D R B 1, D Q B 1 \sim D R B 1, D Q B 1 \sim D Q A 1$ and $B \sim C$ has a substantial probability to be due to type II errors (false negatives). Regarding individual haplotypes, the proportion of haplotypes in significant LD among 1000 simulated sub-samples drawn from human populations is largely under-estimated, being on average 1.5 to 2 times lower than in the original samples (Additional Table S9). Again this suggests that the proportion of individual haplotypes in significant LD is mostly underestimated in chimpanzees, which may explain why it is up to 3 times lower than that observed in humans at most pairs of loci (Additional Table S6). This means that, overall, chimpanzees are expected to display more GLD and more haplotypes in significant LD than observed in our study, which supports our previous conclusion of their greater resemblance to $R G D$ than to $S G D$ populations.

\section{Discussion}

Strong conservation of MHC diversity patterns in humans and chimpanzees

Based on three distinct and complementary statistics describing genetic variation within populations - allelic richness, heterozygosity and nucleotide diversity -, this study has disclosed highly similar patterns of genetic diversity across seven orthologous $M H C$ loci in chimpanzees and humans: overall, both allelic richness and heterozygosity are greater at the three class I loci $A, B, C$ and at $D R B 1$ than at $D Q A 1, D Q B 1$ and $D P B 1$, and nucleotide diversity is greater at loci $D R B 1, D Q A 1, D Q B 1$ and $B$ than at $A, C$ and $D P B 1$ (Fig. 2 and Table 4). In addition, based on both global tests and individual haplotypes' counting, we found similar patterns of linkage disequilibrium across Patr and HLA genes: both highly significant GLD and the highest proportions of individual haplotypes in significant linkage disequilibrium are observed for the same pairs of loci $D Q A 1 \sim D R B 1$, $D Q B 1 \sim D R B 1, D Q B 1 \sim D Q A 1$ and $B \sim C$ (Table 5 and Additional Table S6), which parallels the strong resemblance between Patr and HLA physical maps in chimpanzees and humans, respectively (Fig. 1). These results indicate that the $M H C$ diversity patterns are highly conserved in the human and chimpanzee lineages and that analogous mechanisms drove the evolution of this genomic region in the two species since their divergence from a common ancestor.

\section{Molecular mechanisms generating diversity at MHC genes} In support to the hypothesis that analogous mechanisms drove the evolution of the $M H C$ region in chimpanzees and humans, it has been suggested that the molecular processes generating nucleotide (and hence also allelic) diversity at most $M H C$ loci are similar in both species: new variants would be mainly generated through point mutations at loci $D Q B 1, D Q A 1, C$ and $A$, through recombination and/or gene conversion at loci $D R B 1$ and $B$, and through both kinds of mechanisms at DPB1 [58, 73]. This would partly explain why loci $D R B 1$ and $B$ most often exhibit higher nucleotide and allelic diversity than the other class I and class II loci. Interestingly, chimpanzees contrast with macaques [74, 75] and (to some extent) orangutans [76] and gorillas [77], as the MHC polymorphism of these species (Mamu, Popy and Gogo, respectively) would also evolve through gene duplications at both loci $B$ and $A$.

\section{Signatures of demography on Patr and HLA loci}

Besides the mechanisms generating diversity at the molecular level, both demographic processes and natural selection are known to shape the patterns of populations' genetic diversity at $M H C$ genes, with possible confounding effects [30, 31, 78]. In this regard, it has been suggested that chimpanzees and humans underwent distinct demographic histories [44, 54, 66, 69, 71, 79-83] that probably affected in different ways their $M H C$ profiles [55, 56, 61, 62]. However, demographic evolution has not been uniform in all human populations either [49]. In order to better disentangle the evolutionary mechanisms that drove the evolution of $M H C$ genes in the two species, we thus compared chimpanzees to many different human populations displaying a wide diversity of demographic histories [84] and living in distinct geographical locations - and hence being also submitted to very diverse environmental pressures [23, 85].

As expected, large ranges of genetic diversity values were observed among human populations (Fig. 2). Interestingly, the three genetic diversity indexes - allelic richness, heterozygosity, and nucleotide diversity - appeared to be similar between chimpanzees (especially the wildborn cohorts BPRC $^{\mathrm{wb}}$ and $\mathrm{Kuma}^{\mathrm{wb}}$ ) and the small isolated human populations that likely underwent rapid genetic drift $(R G D)$, regardless of the geographic regions or continents where these human populations lived, and different between chimpanzees and the large outbred 
(SGD) human populations (Fig. 2 and Additional Figure S3). As an example, the very low nucleotide diversity found at the four Patr genes DPB1, DQB1, $C$ and $A$ is comparable to that found at the orthologous HLA genes in Amerindians and Australian Aborigines (Additional Figure S3) as examples of $R G D$ populations. Because neither human populations living in America and Australia nor chimpanzees living in sub-Saharan Africa likely experienced the same pathogenic pressures, comparable demographic histories (i.e. limited population sizes) better explain the similarities than convergent selective effects. Regarding linkage disequilibrium, our simulations indicated that we probably underestimated the amount of GLD and individual haplotypes in significant LD in chimpanzees. This plays in favour of a putative greater resemblance between chimpanzees and $R G D$ (which display high levels of linkage disequilibrium) than between chimpanzees and $S G D$, as a result of genetic drift.

Actually, the idea that Western chimpanzees underwent a substantial reduction in population size has been supported by analysing other parts of the genome. First, studies on both autosomal genes and whole genome sequences [44, 66, 67, 70, 86-88] have indicated that Western chimpanzees are generally less diverse than the other Pan sub-species, which sustains the hypothesis of several past bottlenecks in the former [44, 79]; second, Western chimpanzees' genomic diversity has been found to fall within the average observed for Non-African human populations, which show a much lower genetic diversity than African populations (Fig. 1b of [44]). Therefore, although $M H C$ genes are known to be targets of natural selection, our study reveals that traces of past bottlenecks that impacted non-MHC genes are detectable when analysing the genetic diversity patterns of Patr genes, and more particularly that of the four loci $D P B 1$, $D Q B 1, C$ and $A$.

\section{Signatures of natural selection on Patr and HLA loci}

At the other three $M H C$ loci (DQA1, DRB1 and $B$ ), the genetic diversity observed in Western chimpanzees does not simply mirror that of human populations that likely underwent rapid genetic drift $(R G D)$. Indeed, the nucleotide diversity observed in chimpanzees is either similar to or greater than (significantly at locus $B$ ) that found in human populations with very diverse demographic histories, e.g. in Africa and Europe (Fig. 2, central pane and Additional Figure S3). Furthermore, chimpanzees exhibit both high nucleotide diversity and low heterozygosity compared to human populations at locus $B$, while the reverse (i.e. low nucleotide diversity and high heterozygosity) is found at loci $A$ and $D P B 1$. The differences observed between these genes $(D Q A 1, D R B 1$ and $B)$ and the others (DPB1, $D Q B 1, C$ and $A$ ) is thus probably due to more complex mechanisms involving not only demography (as described above) but also natural selection, i.e. distinct susceptibilities of different Patr genes to pathogenic environments. This is not contradictory with the fact that we did not detect significant departures from selective neutrality for Patr genes in the studied chimpanzee cohorts, as our simulations showed that these results could be due to type II errors.

To better understand how the $M H C$ polymorphism could have evolved in chimpanzees under simultaneous demographic and selective forces, we must first consider which kinds of natural selection may have targeted different Patr genes. According to the scenario that was initially proposed by de Groot et al. [55], a specific mechanism would have affected substantially the $M H C$ genetic profile of chimpanzees, namely a strong selective sweep owing to the action of a viral pathogen (the simian form of $H I V$, i.e. $S I V$ or a related retrovirus) decimating this species $\sim 2$ to 3 million years ago, followed by a second bottleneck in the Western subspecies [44]. As a consequence, many Patr class I alleles would have been lost and the only surviving individuals would have been those carrying alleles providing resistance to the involved pathogen $[55,56]$. This loss of diversity would have specifically affected the Patr- $A$ gene, because at this locus all alleles of a single lineage, $A 2$, were virtually lost, but also the Patr- $B$ and $-C$ genes, based on molecular evidence at intron and $M I C$ regions (see Background above). Actually, as the selective sweep that affected Patr genes had apparently been quite substantial, we would have expected significantly lower (rather than similar) levels of $M H C$ genetic diversity in Western chimpanzees than in small isolated human populations that started to lose diversity much more recently (i.e. at most since modern human populations left their homeland in sub-Saharan Africa). We however have to consider here that chimpanzees had a long time to restore genetic diversity by expanding again demographically after the bottleneck(s) that affected them well before the emergence of modern humans.

Besides a selective sweep, however, balancing selection (in the form of heterozygote advantage) is another mechanism that did affect the evolution of Patr genes. Indeed, $M H C$ genes were found to present strong signals of balancing selection in all great apes' lineages [89]. Moreover, this kind of selection explains the sharing of ancient $M H C$ lineages by humans and chimpanzees at loci $D Q B 1, D Q A 1, D R B 1, C$ and $A[5,16,73,90-92]$. Actually, many works suggest that $M H C$ genes are potential targets of both directional (selective sweep) and balancing selection $[78,93,94]$.

\section{Evolution of Patr genes' diversity: tentative scenarios}

Taking the different evolutionary mechanisms mentioned above into account, i.e. mutational/recombination 
events generating molecular diversity, as well as demographic processes and distinct kinds of natural selection increasing or decreasing the levels of genetic diversity, the results uncovered by the present study support original scenarios for the evolution of Patr genes.

For class I genes, we principally hypothesize that the genetic diversity of Patr-A and Patr-B regenerated when Western chimpanzees expanded demographically (although to a small extent) after the bottlenecks that occurred, first, $\sim 2$ to 3 million years ago in the ancestors of chimpanzees and bonobos [55, 56] and, later on, about 500,000 years ago in the likely differentiated Western chimpanzee subspecies [44]. This idea finds good support in the equivalent amounts of Patr class I nucleotide diversity found in Western (P.t.verus) and Central (P.t.troglodytes) chimpanzees (Fig. 3), in spite of the latter having experienced the least severe population bottleneck among all Pan subspecies [44].

This recovery of genetic variation would have occurred, however, through distinct mechanisms and with distinct intensities at the two loci Patr-A and Patr-B. At Patr-B, recombination and/or gene conversion would have rapidly created new alleles and highly divergent sequences, explaining why chimpanzees, like humans, display higher nucleotide diversity at this locus than at the other class I genes. Asymmetric balancing selection, whereby heterozygotes with more divergent alleles would have an advantage [21, 96] would have also acted on Patr-B, as this type of selection also tends to increase nucleotide diversity [97]. Noteworthy is the fact that particular $H L A-B$ alleles have been positively selected in African human populations in response to Plasmodium falciparum malaria [26] and that functionally similar alleles have recently been identified in bonobos which live in an area with a high prevalence of this parasite [27]. If we assume that common chimpanzees underwent similar responses to pathogens, locus Patr- $B$ (like $H L A-B$ in humans [26]) would have been affected by a (relatively) soft selective sweep whereby several alleles have been positively selected, thus explaining both the high cumulated frequency of three Patr-B alleles (see Supplementary Text) and the high values of heterozygosity and allelic richness found at this locus. By contrast, at Patr-A new variants would have primarily been generated by point mutations that accumulate at slow rates during evolution, which may explain the low nucleotide diversity observed at this locus. Nevertheless, the high heterozygosity found at Patr-A (actually slightly higher than at Patr- $B$ and $H L A-A$ ) suggests that heterozygous advantage also had a substantial effect on this gene after its drastic loss of diversity, possibly as an efficient way to rapidly restore a minimal immune protection despite a slow regeneration of diversity through point mutations. Interestingly, Patr- $A$ molecules display a lower peptide binding repertoire than Patr-B and HLA-A [98], suggesting that they have also evolved a peptide binding site that is more promiscuous $[64,99]$ as a compensation for their severe loss of diversity or that promiscuous alleles were selected preferentially [31]. Finally, the genetic diversity of Patr-A and Patr$B$ might have evolved in concert according to a model of joint asymmetric selection as proposed for $H L A-A$ and $H L A-B[64,100]$, allowing distinct levels of polymorphism to be maintained at the two loci as long as both of them have jointly ensured a sufficient immune protection.

Compared to Patr-A and Patr-B, Patr-C displays a lower level of nucleotide diversity in chimpanzees, like in humans. Knowing that both Patr-C and HLA-C molecules are ligands for killer-cell immunoglobulin-like receptors (KIR) [101, 102], the interaction of HLA and KIR molecules being crucial to regulate the killer function of natural killer cells [103], Patr-C molecules were probably submitted to similar functional constraints as $H L A-C$, resulting in substantial directional and/or purifying selection. However, contrary to Patr-DQB1, for which we suppose the same kinds of selection as for Patr-C (see below), the strong linkage disequilibrium that characterizes the $B \sim C$ loci pair in chimpanzees and humans might have attenuated the opposite effects of balancing and positive/purifying selection impacting loci $B$ and $C$, respectively.

For class II genes, our results also indicate distinct evolutionary histories for the different loci. As $M H C$ class II genes more specifically respond to parasitic and bacterial infections, they would have been less directly impacted by the viral epidemic proposed in $[55,56]$. Moreover, the selection criteria are also less strict as class II genes are generally more promiscuous binders and select longer peptides for binding. Nevertheless, like Patr-A, it is likely that Patr-DRB1 underwent a substantial selective sweep reducing the number of allele lineages, as inferred from its much lower allelic richness compared to Patr-B and in agreement with the apparent loss of all alleles belonging to the DRB1*04 lineage [5]. Such selection would have been mostly independent from that affecting class I genes - i.e. possibly involving other pathogens - since global linkage disequilibrium is not significant between $D R B 1$ and class I genes. Also, because Patr-DRB1 evolves through recombination and/or gene conversion, its putative loss of diversity in the past would have been followed, as proposed above for Patr-B, by a rapid regeneration of nucleotide diversity, which is particularly high at this locus (Fig. 2). Note also that in chimpanzees, $M H C$ class II diversity is particularly high at the haplotype level thanks to inter-locus recombinations despite important loss of variation at single genes due to the past selective sweep [104].

Patr-DQA1 and Patr-DQB1 exhibit contrasting levels of nucleotide diversity and heterozygosity, high for $D Q A 1$ and low for DQB1 (note, however, that Patr- 

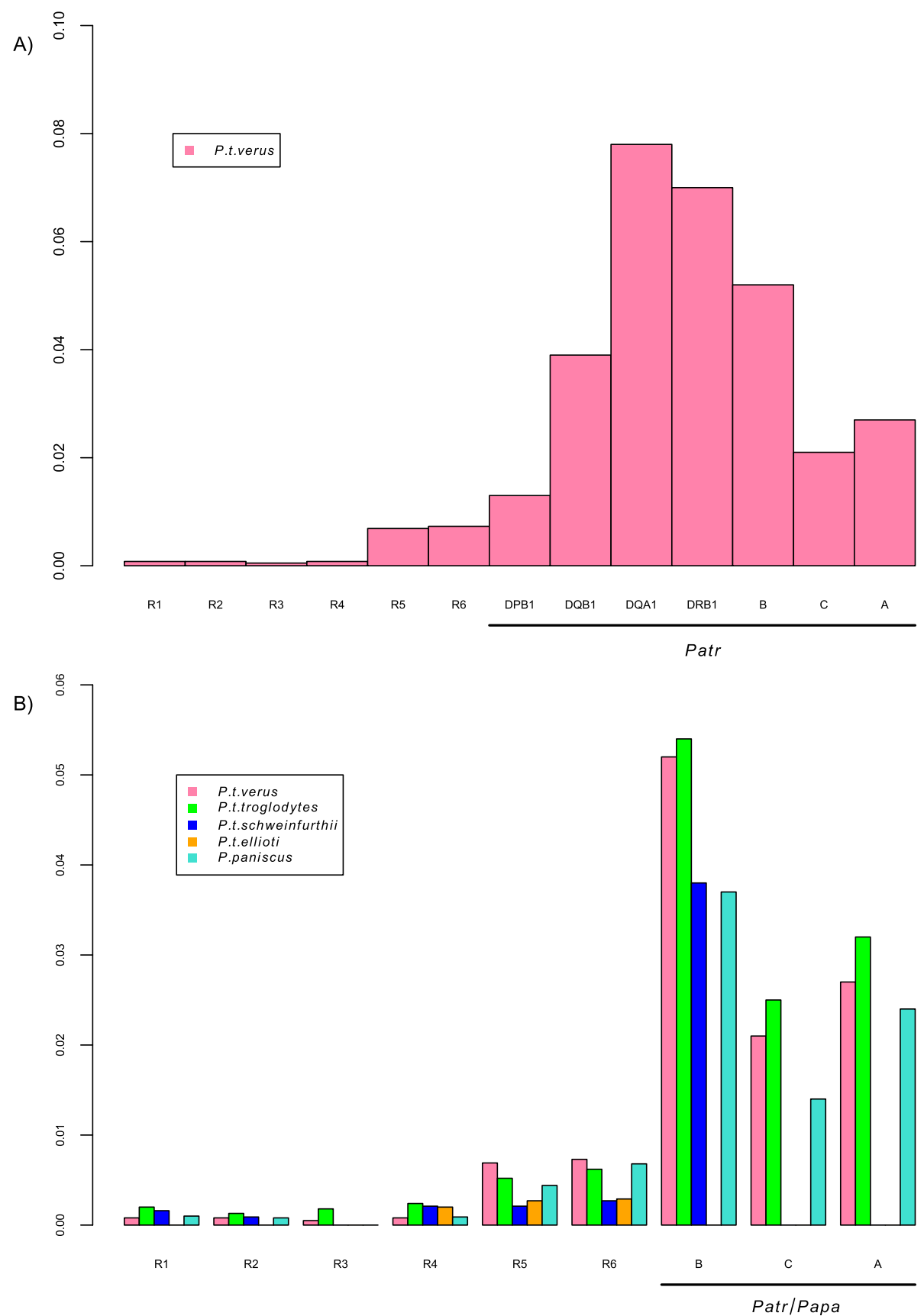

Fig. 3 Nucleotide diversity at MHC loci and other genomic regions in Western chimpanzees (A) and in different sub-species of chimpanzees and bonobos (P. paniscus) (B). R1: Non-coding autosomal regions [66]; R2: Non-coding autosomal regions [67]; R3: Xq13.3 [95]; R4: Non-coding autosomal regions [82]; R5: Mitogenome [82]; R6: Mitogenome [54]; Patr/Papa-B, C, A: average nucleotide diversity for genes Patr/Papa-B, $-C,-A$ : this study, [61, 62]. No data is available for R1, R2 and R3 in P.t.ellioti, for R3 in P.paniscus and for R3 in P.t schweinfurthii. Values are given in Additional Table 510

$D Q A 1$ data were only available for one cohort of chimpanzees, BPRC $^{\mathrm{wb}}$ ), despite the fact that these two genes are in strong linkage disequilibrium and encode the two complementary chains of the Patr-DQ molecules. Among all loci tested, Patr-DQB1 is actually the most divergent to its orthologue in humans for these two indexes (Fig. 2). Studies have stressed the fact that DQ molecules evolve under purifying selection due to strong functional constraints and with a limited dynamic of evolution in both humans and chimpanzees [56, 105]. The low diversity found at Patr-DQB1, with a single allele (DQB1*03:02) reaching a frequency above $60 \%$ in 
the $\mathrm{BPRC}^{\mathrm{wb}}$ cohort (Additional Figure S4), would indicate a stronger constraint on the $\beta$ chain. By contrast, Patr-DQA1 would have evolved by maintaining several alleles (although a limited number, like at $D Q B 1$ ) at more even frequencies, as also observed for $H L A-D Q A 1$ in human populations [21, 106]. Based on our results, we also hypothesize that the very high nucleotide diversity observed at Patr-DQA1 (the highest of all studied loci) results from a molecular evolution mainly characterized by recombination and/or gene conversion rather than point mutations.

Finally, the low nucleotide diversity (and, to a lesser extent, allelic richness) found at Patr-DPB1 is comparable to that observed at $H L A-D P B 1$ in small-sized and isolated populations that likely experienced rapid genetic drift (such as Australian Aborigines and Amerindians), although this is not the case when looking at heterozygosity (Additional Figure S3). These results suggest an effect of balancing selection in the form of heterozygous advantage (explaining the high level of heterozygosity) combined with a slow generation of diversity through point mutations (explaining the low nucleotide diversity falling at the opposite of what is observed for Patr$D Q A 1)$, as suggested for Gogo-DPB1 in gorillas [107]. Interestingly, the low nucleotide diversity observed at DPB1 appears to be rather close to that observed at neutral genomic regions, although the whole Patr region is clearly exceptionally diverse in this respect (Fig. 3 and Additional Table S10).

The main mechanisms that would explain the evolution of the different Patr genes after the ancient bottlenecks that affected Western chimpanzees are illustrated in Fig. 4.

\section{Conclusions}

By revealing similar patterns of genetic diversity and linkage disequilibrium in Western chimpanzees and humans across the main $M H C$ loci, our study suggests that these genes have been shaped by analogous mechanisms in both species despite several million years of independent evolution. This led us to conclude that the $M H C$ region and the evolutionary mechanisms shaping it have been highly conserved in the human and chimpanzee lineages. Our work also uncovered deep similarities between Western chimpanzees and smaller, isolated human populations most likely having undergone rapid genetic drift, independently of their geographic locations and genetic backgrounds, supporting a substantial effect of limited population sizes on $M H C$ evolution in both species. We then proposed plausible scenarios for the molecular evolution of each Patr gene taking into account the strong selective sweep(s) that affected Patr genes after the ancient bottlenecks of Western chimpanzees that, curiously enough, did not substantially deplete their levels of $M H C$ genetic diversity. These scenarios suggest that several Patr genes recovered allelic and/or nucleotide diversity after these bottlenecks thanks to the action of both balancing selection (DRB1, B, A) and rapid generation of polymorphism through recombination and/or gene conversion $(D R B 1, B)$. On the other hand, other loci kept a rather low diversity due to stronger directional or purifying selection and/or a slower process of molecular diversification through point mutations $(D Q B 1, C)$, and some mixed processes also likely occurred (DPB1, DQA1). The possibility to substantially regenerate a high genetic diversity after a bottleneck, as

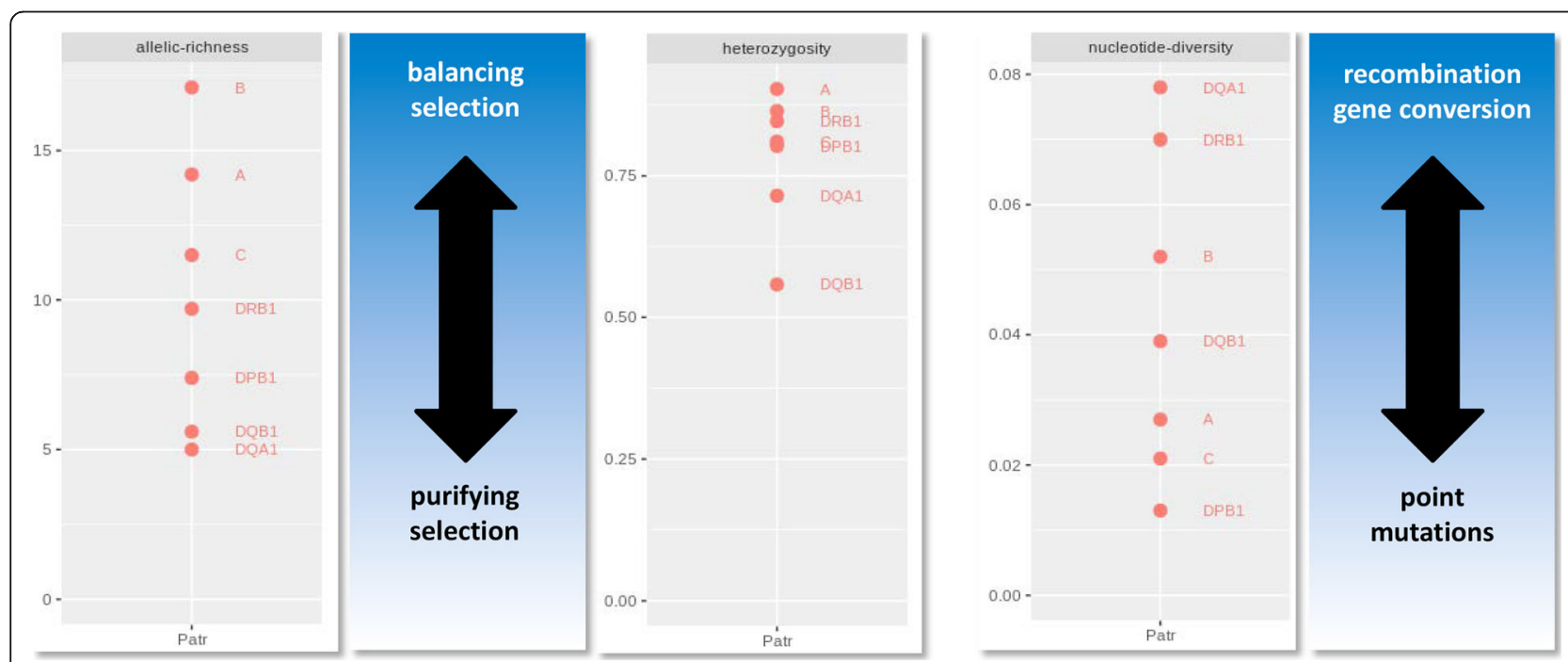

Fig. 4 Schematic representation of the evolutionary mechanisms explaining the genetic diversity observed in Patr genes. For each diversity index, the Patr loci are plotted according to the values given in Table 3 for the pooled cohort of chimpanzees. The pooled cohort includes all cohorts except Texas ${ }^{\mathrm{cb}}$ 
originally proposed for Patr genes in this study, is essential for genes involved in immunity, like those of the $M H C$ complex. Indeed, such a process is likely to restore the potential of a population to resist multiple infectious diseases and may thus be decisive for the long-term survival of critically endangered species like the chimpanzee.

\section{Methods}

\section{Chimpanzee cohorts}

The chimpanzee data include both wild-born $(\mathrm{wb})$ and captive-born (cb) Western chimpanzees (P.t.verus called chimpanzees hereafter) for which Patr analyses were previously published. The available data include four cohorts:

1. BPRC $^{\mathrm{wb}}$, consisting of 29 wild-born individuals captured in Sierra-Leone in the late seventies, who further founded the colony that was originally housed at the Biomedical Primate Research Centre (BPRC) $[16,58,99]$. According to mitochondrial and segregation analyses [108], all individuals appear to be unrelated.

2. Yerkes $^{\mathrm{cb}}$, consisting of 22 captive-born individuals from US institutions $[59,109,110]$. Relatedness between animals is unknown.

3. Texas ${ }^{\mathrm{cb}}$, consisting of 23 captive-born individuals housed in US institutions $[60,111]$. However, contrary to Yerkes ${ }^{\mathrm{cb}}$, this cohort may contain animals from different sub-species and/or hybrid animals (personal communication from the authors of [60]).

4. Kuma ${ }^{\mathrm{wb}}$, consisting of 19 wild-born individuals (of unknown origin, captured in the seventies) who were previously housed in research institutions in Japan and were further retired in the Kumamoto Primate Park, Japan [112-114]. Relatedness between animals is unknown.

We arranged the samples in two ways for the analyses: a) by considering separately the four cohorts defined above; and b) by grouping the individuals from BPRC ${ }^{\mathrm{wb}}$, Yerkes $^{\mathrm{cb}}$ and $\mathrm{Kuma}^{\mathrm{wb}}$ within a single cohort (called the "pooled cohort" hereafter). We did not include Texas ${ }^{\mathrm{cb}}$ in the pooled cohort because of uncertainties regarding the represented sub-species.

The detailed information of each chimpanzee cohort is given in Additional Table S11.

\section{Human populations}

The human data are a subset of 50 to 89 population samples (depending on the locus) taken from the HLA-typed populations analysed in [21]. They represent 10 geographical regions (North Africa, South Africa, North America,
South America, Europe, South-East Asia, North-East Asia, South-West Asia, Australia and Pacific). Based both on a previous paper using most of the same population samples as in this study [100] and on additional ethnological information [50], we defined each population as either RGD (meaning rapid genetic drift) or SGD (meaning slow genetic drift). RGD include small and isolated populations from different continents, mostly Indigenous populations from North and South America, Taiwan, Indonesia, Melanesia and Australia as well as populations from the Saharan region (e.g. Berber speaking) and hunter-gatherers from Central Africa, all other population being classified as SGD. All human populations were analysed separately from each other (i.e. never pooled as a single dataset but considered as multiple populations taken together when reporting the results) in the whole study. The detailed information of each human population sample is given in Additional Table S12.

\section{MHC data}

For both chimpanzee cohorts and human populations, the $M H C$ data consist of multi-locus genotypes (including loci $A, B, C, D R B 1, D Q A 1, D Q B 1$ and/or $D P B 1)$ composed of alleles defined at the 2nd field level of resolution according to the official nomenclatures of the IPD-MHC [115, 116] and IPD-IMGT/HLA [17] databases for Patr and HLA, respectively. At this resolution level, the alleles differ by one or more nucleotide substitutions that change the amino acid sequence of the $M H C$ protein. Moreover, because these data result from exons 2 and 3 (for class I) or exon 2 (for class II) molecular typings, the assessed variation was restricted to the PBR (class I: full exons 2 and 3 sequences; class II: full exon 2 sequences).

A summary of the data used in this study is presented in Table 6.

\section{Statistical analyses Allele frequencies and Hardy-Weinberg equilibrium}

We estimated allele frequencies with an EM algorithm, the Gene-Counting Expectation Maximisation algorithm implemented in [117]. These estimates can be considered as population frequencies if Hardy-Weinberg equilibrium (HWE) is satisfied. We thus tested HWE in all populations by using a Likelihood Ratio Test (LRT) that compares the likelihood of frequencies estimated under HWE to the likelihood of those estimated under an inbreeding model.

\section{Genetic diversity}

We determined genetic diversity within each chimpanzee cohort or human population by using three different statistics: allelic richness ar, expected heterozygosity $h$, and nucleotide diversity $\pi$ : 
Table 6 Summary of the chimpanzee and human population data at Patr and HLA genes, respectively

\begin{tabular}{|c|c|c|c|c|c|c|c|c|}
\hline \multicolumn{2}{|c|}{ Chimpanzees (individual and pooled cohorts) } & DPB1 & $D Q B 1$ & DQA1 & DRB1 & $B$ & C & $A$ \\
\hline \multirow[t]{5}{*}{ N } & $\mathrm{BPRC}^{\mathrm{wb}}$ & 25 & 29 & 29 & 29 & 29 & 29 & 28 \\
\hline & Yerkes $^{\mathrm{cb}}$ & & & & & 22 & 22 & 22 \\
\hline & Texas ${ }^{\mathrm{cb}}$ & & 16 & & 17 & 23 & & 23 \\
\hline & Kumawb & 19 & 19 & & 17 & & & \\
\hline & Pooled cohort ${ }^{a}$ & 44 & 48 & 29 & 46 & 51 & 51 & 50 \\
\hline \multicolumn{2}{|c|}{ Humans (multiple populations) } & $D P B 1$ & $D Q B 1$ & DQA1 & DRB1 & B & C & $A$ \\
\hline \multirow[t]{7}{*}{ k } & Africa & 7 & 19 & 8 & 12 & 11 & 8 & 11 \\
\hline & Europe & 14 & 21 & 15 & 16 & 6 & 3 & 6 \\
\hline & Asia & 9 & 12 & 6 & 33 & 38 & 30 & 36 \\
\hline & N/S America & 11 & 19 & 17 & 18 & 9 & 5 & 9 \\
\hline & Australia & 3 & 2 & 2 & 3 & 4 & 4 & 4 \\
\hline & Pacific & 6 & 6 & 4 & 6 & 5 & 4 & 8 \\
\hline & Other ${ }^{b}$ & 0 & 0 & 0 & 1 & 7 & 5 & 7 \\
\hline \multicolumn{2}{|c|}{$\bar{N}(s . d)$} & $87.8(45.2)$ & $100.6(55.3)$ & 90.7 (45.5) & 105.5 (111.8) & 124 (134.6) & $127.9(141.1)$ & 127.6 (133.6) \\
\hline
\end{tabular}

$\mathrm{N}$ : sample size (in number of individuals); k: number of populations per geographic region; $\bar{N}$ : mean sample size (in number of individuals); s.d.: standard deviation. The order of loci corresponds to their position on the chromosome from centromere (left) to telomere (right)

${ }^{\mathrm{a}}$ does not include Texas ${ }^{\mathrm{cb}}$ (see Text)

${ }^{b}$ Populations were allocated to one of six geographic regions, and we considered an additional category Other for known admixed populations

1. Allelic richness ar was estimated by the number of alleles expected in a population sample of size equal to the rarefaction size $2 n$ (i.e. the size of the smallest sample of $n$ individuals at this locus) [118] as:

$$
a r=\sum_{\mathrm{i}=1}^{\mathrm{k}} 1-\frac{\left(\begin{array}{c}
2 \mathrm{~N}-\mathrm{N}_{\mathrm{i}} \\
2 \mathrm{n}
\end{array}\right)}{\left(\begin{array}{c}
2 \mathrm{~N} \\
2 \mathrm{n}
\end{array}\right)}
$$

where $k$ is the number of alleles in the sample, $2 n$ the rarefaction size and $N_{i}$ the number of occurrences of the $i^{\text {th }}$ allele among the $2 \mathrm{~N}$ sampled genes. Using this index is particularly appropriate when highly polymorphic genes like $M H C$ are studied in samples of small sizes. Rarefaction sizes $(2 n)$ were 50 for $A, 58$ for $B, 56$ for $C, 60$ for $D P B 1,58$ for $D Q A 1,66$ for $D Q B 1$ and 52 for $D R B 1$ when allelic richness was estimated on the pooled cohort of chimpanzees and the different human population samples, and 44 for $A, 44$ for $B, 44$ for $C, 38$ for $D P B 1,58$ for $D Q A 1,32$ for $D Q B 1$ and 34 for $D R B 1$ when the four cohorts of chimpanzees were considered separately.

2. Expected heterozygosity $h$ (equivalent to Nei's gene diversity, [119]) within a sampled population at HWE was computed according to:

$$
h=1-\sum_{\mathrm{i}=1}^{\mathrm{k}} \mathrm{p}_{\mathrm{i}}^{2}
$$

where $k$ is the number of alleles and $p_{i}$ the frequency of the $i$ th allele in the sample. Expected heterozygosity is not necessarily correlated to allelic richness since the latter is only influenced by the number of alleles and not by their frequency; for example, identical allelic richness may be observed in populations showing dissimilar heterozygosity (i.e. high heterozygosity due to the presence of many intermediate frequency alleles, as expected under balancing selection, or low heterozygosity due to the presence of one very frequent and many rare alleles, as expected under purifying selection).

3. Contrary to the expected heterozygosity, nucleotide diversity $\pi$ takes into account the number of nucleotide differences between alleles [119]. To compute this index, a DNA sequence (class I: exon 2 and 3; class II: exon 2) was first assigned to each allele by using the IPD/MHC and IPD/IMGT-HLA resources $[115,116,120]$. Nucleotide diversity was then estimated as:

$$
\pi=\frac{\sum_{i=1}^{k} \sum_{j<i} p_{i} p_{j} d_{i j}}{L}
$$


where $k$ is the number of alleles, $L$ the number of sites in the sequence, $p_{i}$ and $p_{j}$ the frequencies of the $i^{\text {th }}$ and $j^{\text {th }}$ allele in the sample, respectively, and $d_{i j}$ the number of nucleotide differences observed between alleles $i$ and $j$. Nucleotide diversity is not necessarily correlated to expected heterozygosity; for example, identical heterozygosity may be observed in populations showing distinct genetic profiles where alleles are either molecularly very close (i.e. due to their slow diversification through rare point mutations) or molecularly very distant (i.e. due to their rapid diversification through recombination and/or gene conversion).

The three indices described above complement each other as they convey a different information on the genetic diversity observed within a given cohort or population.

\section{Selective neutrality}

To assess whether MHC genes are significantly submitted to selective pressures or behave as neutral markers, we searched for signals of natural selection by applying the Slatkin's version of the Ewens-Watterson selective neutrality test (named EWS test thereafter) based on allele frequencies [121-124] as implemented in [117]. The $p$-values obtained through the resampling process were adjusted for multiple testing using the False Discovery Rate (FDR) method [125]. The tests were done without prior assumptions, thus two-tailed rejection at the 5\% level either occurs above $97.5 \%$ for excess of homozygotes or below $2.5 \%$ for excess of heterozygotes.

\section{Linkage disequilibrium}

As our study explores the genetic diversity at multiple $M H C$ loci, we estimated both global linkage disequilibrium and proportions of haplotypes in significant linkage disequilibrium for all pairs of loci for which data were available. The assessment of global linkage disequilibrium was performed by means of a resampling procedure (named PRS, for Parametric Resampling Schema, hereafter) generating an empirical distribution for a likelihood ratio test (LRT) statistic based on the likelihood of allele and haplotype frequency estimates, the final result being the percentile of the observed LRT statistic (PRS) in the empirical distribution $[106,126]$. Haplotypes in significant linkage disequilibrium were determined by a $x 2$ test (see Supplementary Text).

\section{Genetic distances}

We compared the Patr frequency distributions between each pair of chimpanzee cohorts by computing Prevosti's genetic distances [127] according to:

$$
D_{P, Q}=\frac{1}{2} \sum_{i=1}^{k}\left|p_{i}-q_{i}\right|
$$

where $p_{i}$ and $q_{i}$ represent the frequencies of allele $i$ in populations $P$ and $Q$, respectively. The proportion of shared frequencies between cohorts was then estimated as the complement to 1 of Prevosti's distance given in percentages.

All frequency estimations and statistical analyses based on allele frequencies were performed using the hla-net (www.hla-net.eu) Gene [rate] tools [117]. Arlequin 3.5 [128] and Fstat [129] were used to estimate nucleotide diversity and allelic richness, respectively. When necessary, $p$-values were adjusted using Holm's correction [130].

\section{Computer simulations}

We checked the robustness of our results by controlling for the great discrepancy in sample sizes between chimpanzee cohorts and human populations through computer simulations using a resampling procedure. For each human population sample and each locus, we randomly drew 1000 sub-samples of the same size as the pooled cohort of chimpanzees (i.e. $N=44$ for $D P B 1,48$ for $D Q B 1,29$ for $D Q A 1,46$ for $D R B 1,51$ for $B, 51$ for $C$ and 50 for $A$ ) on which we tested Hardy-Weinberg equilibrium, we estimated the 3 diversity indices, we applied the selective neutrality test and we assessed linkage disequilibrium.

\section{Supplementary information}

Supplementary information accompanies this paper at https://doi.org/10. 1186/s12862-020-01669-6.

Additional file 1: Additional Table S1. Allele frequencies and results of HWE and SEW tests at each Patr locus in the pooled and the four cohorts of chimpanzees.

Additional file 2: Additional Table S2. List of human population samples per HLA locus. For each sample, its population name, it country and region, the population size as well as heterozygosity $(H)$ and nucleotide diversity $(\pi)$, allelic richness (ar) and the results of the Slatkin Ewens-Watterson test of neutrality and Hardy-Weinberg (HW) equilibrium are given. The table presents also the results of the simulations for diversity indices (average value and standard deviation, and the confidence interval at $95 \%$ on the 1000 sub-samples); the results of the simulations for Hardy-Weinberg test (proportion of rejections of HW test in the 1000 sub-samples); the results of the simulations for Ewens-Watterson test (proportion of rejections of Ewens-Watterson test (rejections for both an excess of homozygotes or of heterozygotes) in the 1000 sub-samples).

Additional file 3: Additional Table S3. Genetic diversity at different Patr genes in chimpanzees (multiple cohorts and in the pooled cohort). ar: allelic richness; $H$ : heterozygosity; $\Pi$ : nucleotide diversity; -: data not available; the values of this table were used in Fig. 2.

Additional file 4: Additional Table S4. Test of difference between diversity indexes in chimpanzee cohorts and in human populations subdivided into those that likely followed rapid /or slow genetic drift (RGD and SGD). For each locus, the $p$-value of the Wilcoxon test is given. p-value in bold are significant at 5\% level, and * indicates significant results after correction for the number of loci. 
Additional file 5: Additional Table S5. Results of Global Linkage Disequilibrium (GLD) PRS significance test ${ }^{1}$ between different pairs of $\mathrm{MHC}$ loci in individual chimpanzee cohorts and in the pooled cohort.

Additional file 6: Additional Table S6. Proportion of haplotypes in significant linkage disequilibrium (LD) in chimpanzees (BPRC cohort) and humans (multiple populations, further subdivided into RGD and SGD populations).

Additional file 7: Additional Table S7. Linkage disequilibrium between pairs of alleles in chimpanzees.

Additional file 8: Additional Table S8. Global linkage disequilibrium estimated by PRS test between pairs of loci and proportion of haplotypes in significant linkage disequilibrium for each human population sample.

Additional file 9: Additional Table S9. Results of the simulations on linkage desequilibrium. For each population, the number of simulated samples presenting global linkage disequilibrium (according to the LRT and PRS tests) as well as the average, the standard deviation and the 95\% confidence interval of the proportion of haplotypes in linkage disequilibrium in the simulated samples. The values for the original population sample are given as a reminder.

Additional file 10: Additional Table S10. Nucleotide diversity (П) at Patr loci and other genomic regions in chimpanzees (Pan troglodytes subspecies) and bonobos (Pan paniscus).

Additional file 11: Additional Table S11. List of individuals of the four cohorts of chimpanzees. For each chimpanzee, its name, birth status (wild-born or captive-born), its origin (as defined in the publication), its country of origin (if known), its gender, its sub-species, the publication and the Patr gene studied is given.

Additional file 12: Additional Table S12. List of human population samples per HLA locus. For each sample, population name, country and region of origin and whether the population has likely been submitted to rapid genetic drift are given. Sample size per locus is also given.

Additional file 13: Additional Table S13. Pairs of genotypes per individual and locus.

Additional file 14: Additional Table S14. Number of individuals for each genotype.

Additional file 15. Additional Table S15. Percentages of shared allelic frequencies between chimpanzee cohorts at different Patr loci.

Additional file 16: Additional Table S15. Percentages of shared allelic frequencies between chimpanzee cohorts at different Patr locus.

Additional file 17: Additional Figure S1. Genetic diversity in chimpanzees (pooled cohort) and humans (multiple populations). A) Allelic richness B) heterozygosity, C) nucleotide diversity at each locus under study for the pooled cohort of chimpanzees (in red empty circle) and for the human populations (in blue) represented as violin plots; An average number of $k=70$ (s.d 15.9) human population samples of average size $N=109.2$ (s.d 17.31) were used. The width of the violin varies so as to represent the probability density of the data, the thick blue bar in the centre represents the interquartile range, the thin black line extended from it represents the $95 \%$ confidence intervals, and the green dot is the median. The $\mathrm{MHC}$ loci are presented according to their position on the chromosome from the centromere (left) to the telomere (right).

Additional file 18: Additional Figure S2. Genetic diversity in chimpanzees (pooled cohort) and humans (multiple populations and simulated populations). A) Allelic richness B) heterozygosity, C) nucleotide diversity at each locus under study for the pooled cohort of chimpanzees (in black filled circle) and for the human populations represented as violin plots. Diversity values of each human population sample are plotted as a violin plot in red; Simulated diversity values (1000 samples of the same size per locus as the pooled cohort of chimpanzees) are plotted as a violin plot in blue. The width of the violin varies so as to represent the probability density of the data, the thick blue bar in the centre represents the interquartile range, the thin black line extended from it represents the $95 \%$ confidence intervals, and the green dot is the median. The $\mathrm{MHC}$ loci are presented according to their position on the chromosome from the centromere (left) to the telomere (right).
Additional file 19: Additional Figure S3. Genetic diversity in chimpanzees (multiple cohorts) and humans (multiple populations) averaged by geographic regions. A) Allelic richness B) heterozygosity, C) nucleotide diversity at each locus under study for each cohort of chimpanzees and for the human populations where samples are grouped by geographic regions; The values calculated for each chimpanzee cohort are indicated by filled and unfilled shapes in red for cohorts of wild-born and captive-born chimpanzees, respectively; for the human populations, each region is represented by a triangle of different colour as defined in legend. The MHC loci are presented according to their position on the chromosome from the centromere (left) to the telomere (right).

Additional file 20: Additional Figure S4. Allele frequency distributions for class I and class II loci in the cohorts of chimpanzees including the pooled cohort. 1: locus B, 2: locus C, 3: locus A, 4: locus DPB1, 5: locus DQB1, 6: locus DQA1, and 7: locus DRB1. Alleles are represented by different colours as defined in the legend. Colours in the legend follow the same order as allele frequencies in the plot. Values are in Additional Table S3.

\section{Abbreviations}

MHC: Major Histocompatibility Complex; HLA: Human Leukocyte Antigen; Patr: Name of the $\mathrm{MHC}$ region in chimpanzees, for Pan troglodytes; Papa: Name of the MHC region in bonobos, for Pan paniscus; Mamu: Name of the $\mathrm{MHC}$ region in rhesus monkeys, for Macaca mulatta; Popy: Name of the $\mathrm{MHC}$ region in Bornean orangutans, for Pongo pygmaeus; Gogo: Name of the MHC region in gorillas, for Gorilla gorilla; Myr: Million years; DAA: Divergent Allele Advantage; P. t.: Pan troglodytes; P. p.: Pan paniscus; wb: Wild born; cb: Captive born; BPRC: Biomedical Primate Research Centre; RGD: Rapid Genetic Drift; SGD: Slow Genetic Drift; PBR: Peptide Binding Region; EM: Expectation Maximization; HWE: Hardy-Weinberg Equilibrium; LRT: Likelihood Ratio Test; ar: Allelic richness; h: Heterozygosity; $\pi$ : Nucleotide diversity; EWS: Ewens-Watterson-Slatkin; FDR: False Discovery Rate; PRS: Parametric Resampling Schema; GLD: Global Linkage Disequilibrium; LD: Linkage Disequilibrium; KIR: Killer-cell Immunoglobulin-like Receptors; Kb: Kilobases; s.d: Standard deviation

\section{Acknowledgments}

We warmly thank Stéphane Buhler who kindly gave us access to the formatted genotypes of all human population samples. We are also very grateful to two anonymous reviewers for their constructive comments on a previous version of this manuscript.

\section{Authors' contributions}

CV and ASM conceived and designed the research. CV, JMN and NGG performed Western chimpanzee samples description and coding. CV, JMN and ASM performed research and analysed data, and ESP, NGG and GMD participated in some analyses. CV and ASM wrote the manuscript. JMN, NGG, ESP, GMD and RB participated in drafting of the manuscript. All authors read and approved the final manuscript.

\section{Funding}

This work was supported by the Swiss National Science Foundation, grant \# 310030 188820 to ASM. ESP was supported by Swiss National Science Foundation grant no. 320030_159669. The funding bodies played no role in the design of the study and collection, analysis, and interpretation of data and in writing the manuscript.

\section{Availability of data and materials}

The online version of this article contains supplementary material, which is available to authorized users. Individual chimpanzee details (cohort, number, name, birth status, origin, country of origin, gender, species and publication) alongside MHC genotypes for the chimpanzee cohorts are available in additional files Additional Table S11 and Additional Table S13. Description and results on human populations are in Additional Table S12 and Additional Table S2.

Ethics approval and consent to participate Not applicable. 


\section{Consent for publication}

Not applicable.

\section{Competing interests}

The authors declare there are no competing interests.

\section{Author details}

'Laboratory of Anthropology, Genetics and Peopling History, Department of Genetics and Evolution, Anthropology Unit, University of Geneva, Geneva, Switzerland. ${ }^{2}$ Institute of Genetics and Genomics in Geneva (iGE3), University of Geneva, Geneva, Switzerland. ${ }^{3}$ Comparative Genetics and Refinement, Biomedical Primate Research Centre, 2288, GJ, Rijswijk, The Netherlands.

Received: 27 December 2019 Accepted: 6 August 2020

Published online: 15 September 2020

\section{References}

1. Parham P. The immune system. 4th ed. New York: Garland Science; 2015.

2. Lawlor DA, Ward FE, Ennis PD, Jackson AP, Parham P. HLA-A and B polymorphisms predate the divergence of humans and chimpanzees. Nature. 1988;335(6187):268-71.

3. Klein J, Bontrop RE, Dawkins RL, et al. Nomenclature for the major histocompatibility complexes of different species: a proposal. Immunogenetics. 1990;31(4):217-9.

4. Mayer WE, Jonker M, Klein D, Ivanyi P, van Seventer G, Klein J. Nucleotide sequences of chimpanzee MHC class I alleles: evidence for trans-species mode of evolution. EMBO J. 1988;7(9):2765-74

5. Bontrop RE, Otting N, de Groot NG, Doxiadis GG. Major histocompatibility complex class II polymorphisms in primates. Immunol Rev. 1999;167:339-50.

6. Marsh SG, Albert ED, Bodmer WF, Bontrop RE, Dupont B, Erlich HA, Fernandez-Vina M, Geraghty DE, Holdsworth R, Hurley CK, et al. Nomenclature for factors of the HLA system, 2010. Tissue Antigens. 2010; 75(4):291-455.

7. Adams EJ, Parham P. Genomic analysis of common chimpanzee major histocompatibility complex class I genes. Immunogenetics. 2001;53(3):200-8

8. Adams EJ, Cooper S, Parham P. A novel, nonclassical MHC class I molecule specific to the common chimpanzee. J Immunol. 2001;167(7):3858-69.

9. Geller R, Adams EJ, Guethlein LA, Little AM, Madrigal JA, Parham P. Linkage of Patr-AL to Patr-a and- B in the major histocompatibility complex of the common chimpanzee (Pan troglodytes). Immunogenetics. 2002;54(3):212-5.

10. Andersson G, Larhammar D, Widmark E, Servenius B, Peterson PA, Rask L. Class II genes of the human major histocompatibility complex. Organization and evolutionary relationship of the DR beta genes. J Biol Chem. 1987; 262(18):8748-58.

11. Mnukova-Fajdelova M, Satta Y, O'HUigin C, Mayer WE, Figueroa F, Klein J. Alu elements of the primate major histocompatibility complex. Mamm Genome. 1994:5(7):405-15.

12. Satta Y, Mayer WE, Klein J. Evolutionary relationship of HLA-DRB genes inferred from intron sequences. J Mol Evol. 1996:42(6):648-57.

13. Kriener K, O'HUigin C, Klein J. Alu elements support independent origin of prosimian, platyrrhine, and catarrhine Mhc-DRB genes. Genome Res. 2000; 10(5):634-43.

14. Klein J, Sato A, Nikolaidis N. MHC, TSP, and the origin of species: from immunogenetics to evolutionary genetics. Annu Rev Genet. 2007;41:281304.

15. Doxiadis GG, Hoof I, de Groot N, Bontrop RE. Evolution of HLA-DRB genes. Mol Biol Evol. 2012;29(12):3843-53.

16. de Groot NG, Heijmans CM, de Groot N, Doxiadis GG, Otting N, Bontrop RE. The chimpanzee Mhc-DRB region revisited: gene content, polymorphism, pseudogenes, and transcripts. Mol Immunol. 2009;47(2-3):381-9.

17. Robinson J, Halliwell JA, Hayhurst JD, Flicek P, Parham P, Marsh SG. The IPD and IMGT/HLA database: allele variant databases. Nucleic Acids Res. 2015: 43(Database issue):D423-31.

18. Spurgin LG, Richardson DS. How pathogens drive genetic diversity: MHC, mechanisms and misunderstandings. Proc Biol Sci. 2010;277(1684):979-88.

19. Hedrick PW. Pathogen resistance and genetic variation at MHC loci. Evolution. 2002;56(10):1902-8.

20. Meyer D, Thomson G. How selection shapes variation of the human major histocompatibility complex: a review. Ann Hum Genet. 2001;65:1-26.
21. Buhler S, Sanchez-Mazas A. HLA DNA sequence variation among human populations: molecular signatures of demographic and selective events. PLoS One. 2011;6(2):e14643.

22. Garrigan D, Hedrick PW. Perspective: detecting adaptive molecular polymorphism: lessons from the MHC. Evolution. 2003;57(8):1707-22.

23. Solberg OD, Mack SJ, Lancaster AK, Single RM, Tsai Y, Sanchez-Mazas A, Thomson G. Balancing selection and heterogeneity across the classical human leukocyte antigen loci: a meta-analytic review of 497 population studies. Hum Immunol. 2008;69(7):443-64.

24. Garamszegi LZ. Global distribution of malaria-resistant MHC-HLA alleles: the number and frequencies of alleles and malaria risk. Malar J. 2014;13:349.

25. Hill A, Allsopp C, Kwiatkowski D, Anstey N, Twumasi P, Rowe P, Bennett S, Brewster D, McMichael A, Greenwood B. Common west African HLA antigens are associated with protection from severe malaria. Nature. 1991; 352(6336):595-600.

26. Sanchez-Mazas A, Cerny V, Di D, Buhler S, Podgorna E, Chevallier E, Brunet L, Weber S, Kervaire B, Testi M, et al. The HLA-B landscape of Africa: signatures of pathogen-driven selection and molecular identification of candidate alleles to malaria protection. Mol Ecol. 2017;26(22):6238-52.

27. de Groot NG, Stevens JMG, Bontrop RE. Does the MHC confer protection against malaria in bonobos? Trends Immunol. 2018;39(10):768-71.

28. Alcaide $\mathrm{M}$. On the relative roles of selection and genetic drift in shaping MHC variation. Mol Ecol. 2010;19(18):3842-4.

29. Meyer D, Single RM, Mack SJ, Erlich HA, Thomson G. Signatures of demographic history and natural selection in the human major histocompatibility complex loci. Genetics. 2006;173(4):2121-42.

30. Sutton JT, Nakagawa S, Robertson BC, Jamieson IG. Disentangling the roles of natural selection and genetic drift in shaping variation at MHC immunity genes. Mol Ecol. 2011;20(21):4408-20.

31. Ejsmond MJ, Radwan J. MHC diversity in bottlenecked populations: a simulation model. Conserv Genet. 2011;12(1):129-37.

32. Grogan KE, Sauther ML, Cuozzo FP, Drea CM. Genetic wealth, population health: major histocompatibility complex variation in captive and wild ringtailed lemurs (Lemur catta). Ecol Evol. 2017;7(19):7638-49.

33. Sommer $\mathrm{S}$. The importance of immune gene variability (MHC) in evolutionary ecology and conservation. Front Zool. 2005:2:16

34. de W II, Qurkhuli T, de Groot N, AJM d V-R, van Hooft P, IMA H, HHT P, Bontrop RE, GGM D. Determining Mhc-DRB profiles in wild populations of three congeneric true lemur species by noninvasive methods. Immunogenetics. 2019;71(2):97-107.

35. Radwan J, Biedrzycka A, Babik W. Does reduced MHC diversity decrease viability of vertebrate populations?. Biol Conserv. 2010;143(3):537-44.

36. Reed DH. Albatrosses, eagles and newts, oh my!: exceptions to the prevailing paradigm concerning genetic diversity and population viability? Anim Conserv. 2010;13(5):448-57.

37. Schuster AC, Herde A, Mazzoni CJ, Eccard JA, Sommer S. Evidence for selection maintaining MHC diversity in a rodent species despite strong density fluctuations. Immunogenetics. 2016;68(6-7):429-37.

38. Song X, Zhang P, Huang K, Chen D, Guo S, Qi X, He G, Pan R, Li B. The influence of positive selection and trans-species evolution on DPB diversity in the golden snub-nosed monkeys (Rhinopithecus roxellana). Primates J Primatol. 2016;57(4):489-99.

39. Zhang P, Huang K, Zhang B, Dunn DW, Chen D, Li F, Qi X, Guo S, Li B. High polymorphism in MHC-DRB genes in golden snub-nosed monkeys reveals balancing selection in small, isolated populations. BMC Evol Biol. 2018;18(1):29.

40. Newhouse DJ, Balakrishnan CN. High major histocompatibility complex class I polymorphism despite bottlenecks in wild and domesticated populations of the zebra finch (Taeniopygia guttata). BMC Evol Biol. 2015;15:265.

41. Luo MF, Pan HJ, Liu ZJ, Li M. Balancing selection and genetic drift at major histocompatibility complex class $\|$ genes in isolated populations of golden snub-nosed monkey (Rhinopithecus roxellana). BMC Evol Biol. 2012;12:207.

42. Pechouskova E, Dammhahn M, Brameier M, Fichtel C, Kappeler PM, Huchard E. MHC class II variation in a rare and ecological specialist mouse lemur reveals lower allelic richness and contrasting selection patterns compared to a generalist and widespread sympatric congener. Immunogenetics. 2015; 67(4):229-45.

43. Langergraber KE, Prufer K, Rowney C, Boesch C, Crockford C, Fawcett K, Inoue E, Inoue-Muruyama M, Mitani JC, Muller MN, et al. Generation times in wild chimpanzees and gorillas suggest earlier divergence times in great ape and human evolution. Proc Natl Acad Sci U S A. 2012; 109(39):15716-21. 
44. Prado-Martinez J, Sudmant PH, Kidd JM, Li H, Kelley JL, Lorente-Galdos B, Veeramah KR, Woerner AE, O'Connor TD, Santpere G, et al. Great ape genetic diversity and population history. Nature. 2013;499(7459):471-5.

45. Mellars P. Going East: New Genetic and Archaeological Perspectives on the Modern Human Colonization of Eurasia. Science. 2006;313(5788):796-800.

46. Hublin JJ, Ben-Ncer A, Bailey SE, Freidline SE, Neubauer S, Skinner MM, Bergmann I, Le Cabec A, Benazzi S, Harvati K, et al. New fossils from Jebel Irhoud, Morocco and the pan-African origin of Homo sapiens. Nature. 2017; 546(7657):289-92.

47. Cavalli-Sforza LL. Genes, peoples, and languages. Proc Natl Acad Sci U S A. 1997;94(15):7719-24.

48. Tresset A, Vigne JD. Last hunter-gatherers and first farmers of Europe. Comptes Rendus Biol. 2011;334(3):182-9.

49. Excoffier L, Schneider S. Why hunter-gatherer populations do not show signs of pleistocene demographic expansions. Proc Natl Acad Sci U S A. 1999;96(19):10597-602.

50. Simons GF, Fennig CD. Ethnologue: Languages of the World. 21th ed. Dallas: SIL International. Online version; 2018. http://www.ethnologue.com.

51. McBrearty S, Jablonski NG. First fossil chimpanzee. Nature. 2005;437(7055): 105-8.

52. Kaessmann $\mathrm{H}$, Wiebe $\mathrm{V}$, Paabo $\mathrm{S}$. Extensive nuclear DNA sequence diversity among chimpanzees. Science. 1999;286(5442):1159-62.

53. Wegmann D, Excoffier L. Bayesian inference of the demographic history of chimpanzees. Mol Biol Evol. 2010;27(6):1425-35.

54. Hvilsom C, Carlsen F, Heller R, Jaffre N, Siegismund HR. Contrasting demographic histories of the neighboring bonobo and chimpanzee. Primates. 2014;55(1):101-12.

55. de Groot NG, Otting N, Doxiadis GM, Balla-Jhagjhoorsingh S, Heeney JL, van Rood J, Gagneux P, Bontrop RE. Evidence for an ancient selective sweep in the MHC class I gene repertoire of chimpanzees. Proc Natl Acad Sci. 2002; 99(18):11748-53.

56. de Groot NG, Heijmans CMC, De Groot N, Otting N, De Vos-Rouweler AJM, Remarque EJ, Bonhomme M, Doxiadis GG, Crouau-Roy B, Bontrop RE. Pinpointing a selective sweep to the chimpanzee $\mathrm{MHC}$ class I region by comparative genomics. Mol Ecol. 2008;17(8):2074-88.

57. McAdam SN, Boyson JE, Liu X, Garber TL, Hughes AL, Bontrop RE, Watkins DI. Chimpanzee MHC class I A locus alleles are related to only one of the six families of human A locus alleles. J Immunol. 1995;154(12):6421-9.

58. de Groot NG, Otting N, Argüello R, Watkins DI, Doxiadis GG, Madrigal JA, Bontrop RE. Major histocompatibility complex class I diversity in a west African chimpanzee population: implications for HIV research. Immunogenetics. 2000;51(6):398-409.

59. Adams EJ, Cooper S, Thomson G, Parham P, Adams E. Common chimpanzees have greater diversity than humans at two of the three highly polymorphic MHC class I genes. Immunogenetics. 2000;51(6):410-24.

60. Caggiari L, Simula MP, Marzotto A, Shiina M, Rehermann B, De Re V. Identification of novel chimpanzee MHC class I and II alleles using an improved sequence-based typing strategy. Hum Immunol. 2006;67(1-2):63-72.

61. Maibach V, Hans JB, Hvilsom C, Marques-Bonet T, Vigilant L. MHC class diversity in chimpanzees and bonobos. Immunogenetics. 2017;69(10): 661-76.

62. Wroblewski EE, Norman PJ, Guethlein LA, Rudicell RS, Ramirez MA, Li Y, Hahn BH, Pusey AE, Parham P. Signature patterns of MHC diversity in three Gombe communities of wild chimpanzees reflect fitness in reproduction and immune defense against SIVcpz. PLoS Biol. 2015;13(5): e1002144.

63. de Groot NG, Heijmans CMC, Helsen P, Otting N, Pereboom Z, Stevens JMG, Bontrop RE. Limited MHC class I intron 2 repertoire variation in bonobos Immunogenetics. 2017;69(10):677-88.

64. de Groot NG, Bontrop RE. The HIV-1 pandemic: does the selective sweep in chimpanzees mirror humankind's future? Retrovirology. 2013;10:53.

65. Kulski JK, Shiina T, Anzai T, Kohara S, Inoko H. Comparative genomic analysis of the MHC: the evolution of class I duplication blocks, diversity and complexity from shark to man. Immunol Rev. 2002;190:95-122.

66. Fischer A, Pollack J, Thalmann O, Nickel B, Paabo S. Demographic history and genetic differentiation in apes. Curr Biol. 2006;16(11):1133-8.

67. Yu N, Jensen-Seaman MI, Chemnick L, Kidd JR, Deinard AS, Ryder O, Kidd KK, Li WH. Low nucleotide diversity in chimpanzees and bonobos. Genetics. 2003;164(4):1511-8.

68. Becquet C, Patterson N, Stone AC, Przeworski M, Reich D. Genetic structure of chimpanzee populations. PLoS Genet. 2007;3(4):e66.
69. Kaessmann H, Wiebe V, Weiss G, Paabo S. Great ape DNA sequences reveal a reduced diversity and an expansion in humans. Nat Genet. 2001;27(2): 155-6.

70. Bataillon T, Duan J, Hvilsom C, Jin X, Li Y, Skov L, Glemin S, Munch K, Jiang $T$, Qian $Y$, et al. Inference of purifying and positive selection in three subspecies of chimpanzees (Pan troglodytes) from exome sequencing. Genome Biol Evol. 2015;7(4):1122-32.

71. Kuhlwilm M, de Manuel M, Nater A, Greminger MP, Krützen M, MarquesBonet T. Evolution and demography of the great apes. Curr Opin Genet Dev. 2016:41:124-9.

72. IUCN: The IUCN Red List of Threatened Species. Version 2018-1. In. Downloaded on 22 August 2018; 2018. http://www.iucnredlist.org.

73. Otting N, Doxiadis GG, Versluis L, de Groot NG, Anholts J, Verduin W, Rozemuller E, Claas F, Tilanus MG, Bontrop RE. Characterization and distribution of Mhc-DPB1 alleles in chimpanzee and rhesus macaque populations. Hum Immunol. 1998;59(10):656-64.

74. Doxiadis GG, de Groot N, Otting N, Blokhuis JH, Bontrop RE. Genomic plasticity of the $\mathrm{MHC}$ class I a region in rhesus macaques: extensive haplotype diversity at the population level as revealed by microsatellites. Immunogenetics. 2011;63(2):73-83.

75. Doxiadis GG, de Groot N, Otting N, de Vos-Rouweler AJM, Bolijn MJ, Heijmans CMC, de Groot NG, van der Wiel MKH, Remarque EJ, Vangenot C, et al. Haplotype diversity generated by ancient recombination-like events in the MHC of Indian rhesus macaques. Immunogenetics. 2013;65(8):569-84.

76. de Groot NG, Heijmans CM, van der Wiel MK, Blokhuis JH, Mulder A, Guethlein LA, Doxiadis GG, Claas FH, Parham P, Bontrop RE. Complex MHC Class I Gene Transcription Profiles and Their Functional Impact in Orangutans. J Immunol. 2016;196(2):750-8.

77. Hans JB, Bergl RA, Vigilant L. Gorilla MHC class I gene and sequence variation in a comparative context. Immunogenetics. 2017;69(5):303-23.

78. Sanchez-Mazas A, Cerny V, Buhler S, Podgorna E, Chevallier E, Brunet L, Weber S, Kervaire B, Testi M, Andreani M, et al. The HLA-B landscape of Africa: Signatures of pathogen-driven selection and molecular identification of candidate alleles to malaria protection. Mol Ecol. 2017;26(22):6238-52.

79. de Manuel M, Kuhlwilm M, Frandsen P, Sousa VC, Desai T, Prado-Martinez J, Hernandez-Rodriguez J, Dupanloup I, Lao O, Hallast P, et al. Chimpanzee genomic diversity reveals ancient admixture with bonobos. Science. 2016; 354(6311):477-81.

80. Lobon I, Tucci S, de Manuel M, Ghirotto S, Benazzo A, Prado-Martinez J, Lorente-Galdos B, Nam K, Dabad M, Hernandez-Rodriguez J, et al. Demographic history of the genus Pan inferred from whole mitochondrial genome reconstructions. Genome Biol Evol. 2016;8(6):2020-30.

81. Gagneux P, Wills C, Gerloff U, Tautz D, Morin PA, Boesch C, Fruth B, Hohmann G, Ryder OA, Woodruff DS. Mitochondrial sequences show diverse evolutionary histories of African hominoids. Proc Natl Acad Sci U S A. 1999;96(9):5077-82.

82. Fischer A, Prufer K, Good JM, Halbwax M, Wiebe V, Andre C, Atencia R, Mugisha L, Ptak SE, Paabo S. Bonobos fall within the genomic variation of chimpanzees. PLoS One. 2011;6(6):e21605.

83. Gonder MK, Locatelli S, Ghobrial L, Mitchell MW, Kujawski JT, Lankester FJ, Stewart CB, Tishkoff SA. Evidence from Cameroon reveals differences in the genetic structure and histories of chimpanzee populations. Proc Natl Acad Sci U S A. 2011;108(12):4766-71.

84. Sanchez-Mazas A, Fernandez-Vina M, Middleton D, Hollenbach JA, Buhler S, Di D, Rajalingam R, Dugoujon JM, Mack SJ, Thorsby E. Immunogenetics as a tool in anthropological studies. Immunology. 2011;133(2):143-64.

85. Currat M, Poloni ES, Sanchez-Mazas A. Human genetic differentiation across the strait of Gibraltar. BMC Evol Biol. 2010;10:237.

86. Becquet C, Przeworski M. A new approach to estimate parameters of speciation models with application to apes. Genome Res. 2007;17(10): 1505-19.

87. The Chimpanzee Sequencing and Analysis Consortium. Initial sequence of the chimpanzee genome and comparison with the human genome. Nature. 2005:437(7055):69-87.

88. Vangenot C, Gagneux P, de Groot NG, Baumeyer A, Mouterde M, CrouauRoy B, Darlu P, Sanchez-Mazas A, Sabbagh A, Poloni ES. Humans and Chimpanzees Display Opposite Patterns of Diversity in Arylamine NAcetyltransferase Genes. G3 (Bethesda). 2019;9(7):2199-224.

89. Cagan A, Theunert C, Laayouni H, Santpere G, Pybus M, Casals F, Prufer K, Navarro A, Marques-Bonet T, Bertranpetit J, et al. Natural selection in the great apes. Mol Biol Evol. 2016;33(12):3268-83. 
90. Bontrop RE. Comparative genetics of MHC polymorphisms in different primate species: duplications and deletions. Hum Immunol. 2006;67(6): 388-97.

91. Doxiadis GG, de Groot N, de Groot NG, Doxiadis II, Bontrop RE. Reshuffling of ancient peptide binding motifs between HLA-DRB multigene family members: old wine served in new skins. Mol Immunol. 2008;45(10):2743-51.

92. Otting N, de Groot NG, Doxiadis GG, Bontrop RE. Extensive Mhc-DQB variation in humans and non-human primate species. Immunogenetics. 2002:54(4):230-9.

93. Sanchez-Mazas A, Lemaître J-F, Currat M. Distinct evolutionary strategies of human leucocyte antigen loci in pathogen-rich environments. Philos Trans R Soc B Biol Sci. 2012;367(1590):830-9.

94. Vassilakos D, Natoli A, Dahlheim M, Hoelzel AR. Balancing and directional selection at exon-2 of the MHC DQB1 locus among populations of odontocete cetaceans. Mol Biol Evol. 2009;26(3):681-9.

95. Kaessmann H. Human and Chimpanzee Nucleotide Diversity. In: Encyclopedia of Life Sciences (ELS). Chichester: Wiley; 2008.

96. Bronson PG, Mack SJ, Erlich HA, Slatkin M. A sequence-based approach demonstrates that balancing selection in classical human leukocyte antigen (HLA) loci is asymmetric. Hum Mol Genet. 2013;22(2):252-61.

97. Satta Y. Effects of intra-locus recombination of HLA polymorphism. Hereditas. 1997;127(1-2):105-12.

98. van Deutekom HW, Hoof I, Bontrop RE, Kesmir C. A comparative analysis of viral peptides presented by contemporary human and chimpanzee MHC class I molecules. J Immunol. 2011;187(11):5995-6001.

99. de Groot NG, Heijmans CMC, Zoet YM, de Ru AH, Verreck FA, van Veelen PA, Drijfhout JW, Doxiadis GG, Remarque EJ, Doxiadis IIN, et al. AIDSprotective $\mathrm{HLA}-\mathrm{B}^{*} 27 / \mathrm{B}^{*} 57$ and chimpanzee $\mathrm{MHC}$ class I molecules target analogous conserved areas of HIV-1/SIVcpz. Proc Natl Acad Sci. 2010;107(34): 15175-80.

100. Buhler S, Nunes JM, Sanchez-Mazas A. HLA class I molecular variation and peptide-binding properties suggest a model of joint divergent asymmetric selection. Immunogenetics. 2016;68(6-7):401-16.

101. Parham P, Moffett A. Variable NK cell receptors and their MHC class I ligands in immunity, reproduction and human evolution. Nat Rev Immunol. 2013; 13(2):133-44.

102. Wroblewski EE, Parham P, Guethlein LA. Two to tango: co-evolution of hominid natural killer cell receptors and MHC. Front Immunol. 2019;10:177.

103. Carrington M, Norman PJ. The KIR gene cluster: Bethesda (MD): National Center for biotechnology information (US); 2003.

104. Otting N, de Groot NG, Bontrop RE. Limited MHC class II gene polymorphism in the west African chimpanzee is distributed maximally by haplotype diversity. Immunogenetics. 2019;71(1):13-23.

105. Kwok WW, Nepom GT, Raymond FC. HLA-DQ polymorphisms are highly selective for peptide binding interactions. J Immunol. 1995;155(5):2468-76.

106. Sanchez-Mazas A, Buhler S, Nunes JM. A new HLA map of Europe: regional genetic variation and its implication for peopling history, disease-association studies and tissue transplantation. Hum Hered. 2013;76(3-4):162-77.

107. Hans JB, Haubner A, Arandjelovic M, Bergl RA, Fünfstück T, Gray M, Morgan DB, Robbins MM, Sanz C, Vigilant L. Characterization of MHC class II B polymorphism in multiple populations of wild gorillas using non-invasive samples and nextgeneration sequencing. Am J Primatol. 2015;77(11):1193-206.

108. de Groot NG, Garcia CA, Verschoor EJ, Doxiadis GG, Marsh SG, Otting N, Bontrop RE. Reduced MIC gene repertoire variation in west African chimpanzees as compared to humans. Mol Biol Evol. 2005;22(6):1375-85.

109. Cooper S, Adams EJ, Wells RS, Walker CM, Parham P. A major histocompatibility complex class I allele shared by two species of chimpanzee. Immunogenetics. 1998:47(3):212-7.

110. Shiina T, Ota M, Shimizu S, Katsuyama Y, Hashimoto N, Takasu M, Anzai T, Kulski JK, Kikkawa E, Naruse T, et al. Rapid evolution of major histocompatibility complex class I genes in Primates generates new disease alleles in humans via hitchhiking diversity. Genetics. 2006:173(3):1555-70.

111. Mizukoshi E, Nascimbeni M, Blaustein JB, Mihalik K, Rice CM, Liang TJ, Feinstone SM, Rehermann B. Molecular and immunological significance of chimpanzee major histocompatibility complex haplotypes for hepatitis C virus immune response and vaccination studies. J Virol. 2002;76(12):6093-103.

112. Bak EJ, Ishii Y, Omatsu T, Kyuwa S, Hayasaka I, Yoshikawa Y. Sequence analysis of the MHC class II DPB1 gene in chimpanzees (Pan troglodytes). Int J Immunogenet. 2005;32(3):187-92.
113. Bak EJ, Ishii Y, Omatsu T, Kyuwa S, Tetsuya T, Hayasaka I, Yoshikawa Y. Identification and analysis of MHC class II DRB1 (Patr-DRB1) alleles in chimpanzees. Tissue Antigens. 2006;67(2):134-42.

114. Bak EJ, Ishii Y, Omatsu T, Kyuwa S, Tanoue T, Hayasaka I, Yoshikawa Y. Sequence analysis of major histocompatibility complex class-II DQB1 (PatrDQB1) alleles in chimpanzees by polymerase chain reaction-based methods. Hum Immunol. 2006;67(8):655-63.

115. Maccari G, Robinson J, Ballingall K, Guethlein LA, Grimholt U, Kaufman J, Ho CS, de Groot NG, Flicek P, Bontrop RE, et al. IPD-MHC 2.0: an improved inter-species database for the study of the major histocompatibility complex. Nucleic Acids Res. 2017:45(D1):D860-d864.

116. de Groot NG, Otting N, Maccari G, Robinson J, Hammond JA, Blancher A, Lafont BAP, Guethlein LA, Wroblewski EE, Marsh SGE, et al. Nomenclature report 2019: major histocompatibility complex genes and alleles of great and small ape and old and New World monkey species. Immunogenetics. 2020;72(1-2):25-36.

117. Nunes JM. Using uniformat and gene [rate] to analyze data with ambiguities in population genetics. Evol Bioinformatics Online. 2015; 11(Suppl 2):19-26.

118. El Mousadik A, Petit RJ. High level of genetic differentiation for allelic richness among populations of the argan tree [Argania spinosa (L.) Skeels] endemic to Morocco. Theor Appl Genet. 1996;92(7):832-9.

119. Nei M. Molecular evolutionary genetics: Columbia University press; 1987

120. de Groot NG, Otting N, Robinson J, Blancher A, Lafont BA, Marsh SG, O'Connor DH, Shiina T, Walter L, Watkins DI, et al. Nomenclature report on the major histocompatibility complex genes and alleles of great ape, old and New World monkey species. Immunogenet. 2012;64(8):615-31.

121. Ewens WJ. The sampling theory of selectively neutral alleles. Theor Popul Biol. 1972;3(1):87-112.

122. Slatkin M. An exact test for neutrality based on the Ewens sampling distribution. Genet Res. 1994:64(1):71-4.

123. Slatkin M. A correction to the exact test based on the Ewens sampling distribution. Genet Res. 1996:68(3):259-60.

124. Watterson GA. The homozygosity test of neutrality. Genetics. 1978;88(2): 405-17.

125. Benjamini Y, Hochberg Y. Controlling the false discovery rate: a practical and powerful approach to multiple testing. J R Stat Soc Ser B Methodol. 1995;57(1):289-300.

126. Buhler S, Nunes JM, Nicoloso G, Tiercy JM, Sanchez-Mazas A. The heterogeneous HLA genetic makeup of the Swiss population. PLoS One. 2012;7(7):e41400.

127. Prevosti A, Ocana J, Alonso G. Distances between populations ofDrosophila subobscura, based on chromosome arrangement frequencies. Theor Appl Genet. 1975;45(6):231-41.

128. Excoffier $L$, Lischer HE. Arlequin suite ver 3.5: a new series of programs to perform population genetics analyses under Linux and windows. Mol Ecol Resour. 2010;10(3):564-7.

129. Goudet J. FSTAT, a program to estimate and test gene diversities and fixation indices (version 2.9.3); 2001

130. Holm S. A simple sequentially rejective multiple test procedure. Scand J Stat. 1979;6:65-70

\section{Publisher's Note}

Springer Nature remains neutral with regard to jurisdictional claims in published maps and institutional affiliations.

Ready to submit your research? Choose BMC and benefit from:

- fast, convenient online submission

- thorough peer review by experienced researchers in your field

- rapid publication on acceptance

- support for research data, including large and complex data types

- gold Open Access which fosters wider collaboration and increased citations

- maximum visibility for your research: over $100 \mathrm{M}$ website views per year

At $\mathrm{BMC}$, research is always in progress.

Learn more biomedcentral.com/submission 